Pacific

Journal of

Mathematics

SUMMATION OF FORMAL SOLUTIONS OF A CLASS OF LINEAR DIFFERENCE EQUATIONS

B.L.J. Braaksma, B.F. Faber, and G.K. Immink 


\title{
SUMMATION OF FORMAL SOLUTIONS OF A CLASS OF LINEAR DIFFERENCE EQUATIONS
}

\author{
B.L.J. BraAksma, B.F. FABER, And G.K. IMminK
}

Dedicated to the memory of W.A. Harris, Jr

\begin{abstract}
We consider difference equations $y(s+1)=A(s) y(s)$, where $A(s)$ is an $n \times n$-matrix meromorphic in a neighborhood of $\infty$ with $\operatorname{det} A(s) \not \equiv 0$. In general, the formal fundamental solutions of this equation involve gamma-functions which give rise to the critical variable $s \log s$ and a level $1^{+}$. We show that, under a mild condition, formal fundamental matrices of the equation can be summed uniquely to analytic fundamental matrices represented asymptotically by the formal fundamental solution in appropriate domains.

The method of proof is analogous to a method used to prove multi-summability of formal solutions of ODE's. Starting from analytic lifts of the formal fundamental matrix in half planes, we construct a sequence of increasingly precise quasi-functions, each of which is determined uniquely by its predecessor.
\end{abstract}

\section{Introduction.}

This paper is concerned with summability of formal solutions of linear homogeneous difference equations. We consider the system

$$
y(s+1)=\mathrm{A}(s) y(s),
$$

where $s$ is a complex variable, $y(s) \in \mathbf{C}^{n}$, and $\mathrm{A}(s)$ an $n \times n$-matrix, meromorphic at infinity, $\operatorname{det} A(s) \not \equiv 0$. For some $p \in \mathbf{N}$ Equation (1.1) has a formal fundamental matrix solution of the form

$$
\hat{\mathrm{Y}}(s)=\hat{\mathrm{H}}(s) s^{\Lambda s} e^{\mathrm{G}(s)} s^{\mathrm{L}},
$$

with $\hat{\mathrm{H}}(s) \in \operatorname{End}\left(n, \mathbf{C}\left[\left[s^{-1 / p}\right]\right]\right)$, det $\hat{\mathrm{H}}(s) \not \equiv 0, \Lambda=\bigoplus_{j=1}^{m} \lambda_{j} \mathrm{l}_{j}$ where $\lambda_{j} \in \frac{1}{p} \mathbf{Z}$ and $\mathrm{I}_{j}$ is the $n_{j} \times n_{j}$-identity matrix, $\mathrm{G}(s)=\bigoplus_{j=1}^{m} g_{j}(s) \mathrm{I}_{j}$ where $g_{j}(s) \equiv 0$ or $g_{j}(s)$ is a polynomial in $s^{1 / p}$ of degree at most $p$ with $g_{j}(0)=0$, and $\mathrm{L}=\bigoplus_{j=1}^{m} \mathrm{~L}_{j}, \mathrm{~L}_{j}=c_{j} \mathrm{l}_{j}+\mathrm{N}_{j}$ with $c_{j} \in \mathbf{C}$ and $\mathrm{N}_{j}$ an $n_{j} \times n_{j}$-nilpotent matrix, and with $n_{1}+n_{2}+\cdots+n_{m}=n$. 
The purpose of this paper is to sum the entries of $\hat{\mathrm{H}}(s)$ on certain unbounded domains $D$ in order to obtain uniquely characterizable analytic fundamental matrix solutions

$$
\begin{aligned}
& \mathrm{Y}(s)=\mathrm{H}(s) s^{\Lambda s} e^{\mathrm{G}(s)} s^{\mathrm{L}}, \text { with } \\
& \mathrm{H}(s) \sim \hat{\mathrm{H}}(s), s \rightarrow \infty \text { on } D .
\end{aligned}
$$

Any solution of (1.1) on $D$ can be written as $\mathrm{Y}(s) P(s)$ where $P(s)$ is a 1-periodic $\mathbf{C}^{n}$-valued function.

If the factor $s^{\Lambda s}$ does not appear in the formal fundamental matrix, i.e., if all $\lambda_{j}$ 's vanish, the formal fundamental matrix resembles that of a homogeneous linear differential system. Formal power series solutions of meromorphic differential equations can be summed by means of a method known as multisummation. With such an equation one can associate so-called 'levels', positive rational numbers $k_{1}, \ldots, k_{r}$, and corresponding 'critical variables' $s^{k_{1}}, \ldots, s^{k_{r}}$, which play a crucial part in the summation process. Multi-summation is a particular case of accelero-summation (see [Eca87]), involving only elementary accelerations. There exist various equivalent definitions of multisummability (see Definition 2). It can be formulated in terms of Borel and Laplace transforms (cf. [MR91]), or in a more abstract way (cf. [MR92]). In [Bal94] Balser presented yet another definition. Multisummability of solutions of both linear and nonlinear meromorphic differential equations has been proved both by using Borel-Laplace techniques (see [Bra91] and [Bra92]) and in a way based on the definition of Malgrange and Ramis (see [BBRS91], [RS94], [Bal94], [Tov96], and [BIS]).

Two of the most important features that distinguish linear difference equations from linear differential equations are:

(i) The solution space of a homogeneous linear difference equation is linear over the 1-periodic functions instead of $\mathbf{C}$-linear as in the case of homogeneous linear differential equations.

(ii) The occurrence of the factor $s^{\Lambda s}$, that does not appear in formal solutions of differential equations.

If the factor $s^{\Lambda s}$ does not appear in the formal fundamental matrix, or, more generally, if all $\lambda_{j}$ 's are equal, then all entries of $\hat{\mathrm{H}}(s)$ are multisummable in all but at most a countably infinite number of directions. This was shown in [BF96] by means of Borel-Laplace techniques in the spirit of the work of Ecalle [Eca85]. With the same techniques multisummability of formal solutions of a class of non-linear difference equations was proved there.

If not all $\lambda_{j}$ 's are equal, some of the entries of $\hat{\mathrm{H}}(s)$ may not be multisummable in any direction. This is due to the fact that, in this case, one of the critical variables is $s \log s$, which is not a rational power of $s$.

Following Ecalle (cf. [Eca85]), one might set out to sum the formal solutions by accelero-summation, using Borel and Laplace transforms. For a 
particular class of linear difference equations, accelero-summability of the formal solutions was established in [Imm]. Ecalle's method involves the study of a convolution equation, obtained from the equation satisfied by $\hat{H}$ by means of a formal Borel transformation in the variable $s \log s$, which does not look very inviting. In the present paper we take a different approach, similar to the method employed in [BIS] to sum formal solutions of linear differential equations (cf. Theorem 13). Our starting point is the "main asymptotic existence theorem' for difference equations (Theorem 6), which says that $\hat{\mathrm{H}}(s)$ can be lifted on half planes in $\mathbf{C}_{\infty}$, bounded by the real or imaginary axis, to an analytic matrix $\mathrm{H}(s)$ such that (1.3) defines an analytic fundamental matrix $\mathrm{Y}(s)$ of the difference equation. With the equation we associate certain levels $0<k_{1}<\cdots<k_{r}=1$, that can be extracted from the formal fundamental solution, as well as a level $1^{+}$if not all $\lambda_{j}$ 's are equal (see Definitions 3 and 5). We choose a covering of a neighbourhood of $\infty$ in $\mathbf{C}_{\infty}$ by appropriate half planes and, on each half plane a fundamental system of (1.1) represented asymptotically by the formal fundamental system (1.2). In several steps, modifying the solutions by exponentially small functions of increasing order at each subsequent step, we construct a sequence of so-called $k_{j}$-precise quasi-functions, $j=1, \ldots, r$. If the equation does not possess a level $1^{+}$, this procedure yields the multi-sum, or $\left(k_{1}, \ldots, k_{r}\right)$-sum of the formal solution (Theorem 13).

If the equation does possess level $1^{+}$, the final step is more delicate than the preceding ones. This is due to the relative 'closeness' of the levels 1 and $1^{+}$and the transcendental nature of the critical variable $s \log s$. In order to end up with a unique sum, we need to consider domains that are strictly smaller than half planes, but sufficiently large to exclude the existence of flat solutions of the difference equation satisfied by $\hat{H}$, with a dominant factor of the form $s^{\left(\lambda_{i}-\lambda_{j}\right) s}$, with $\lambda_{i} \neq \lambda_{j}$. Here we shall consider domains of the type $\left\{s \in \mathbf{C}_{\infty} \mid \arg s \in((h-1) \pi,(h+1) \pi),(-1)^{h} \Re\{s(\log s+i \theta)\}>1\right\}$, with $\theta \in \mathbf{R}, h \in \mathbf{Z}$ (cf. Figures 2-5). On the union of two such domains with the same $h$ we can define a sum $\mathrm{H}(s)$ of $\hat{\mathrm{H}}(s)$ if a certain generic condition is satisfied (cf. Section 7). By means of (1.3) we obtain a unique analytic fundamental matrix of the difference equation (Theorem 18).

In order to illustrate the particular properties of difference equations with level $1^{+}$, we end this introduction with a simple example.

Example 1. Consider the equation

$$
h(s+1)-a s^{-1} h(s)=s^{-1} \text { with } a \in \mathbf{R}, a>0
$$

which can be transformed into the matrix equation

$$
\left(\begin{array}{l}
y_{1} \\
y_{2}
\end{array}\right)(s+1)=\left(\begin{array}{cc}
a / s & 1 / s \\
0 & 1
\end{array}\right)\left(\begin{array}{l}
y_{1} \\
y_{2}
\end{array}\right)(s) .
$$


(1.4) belongs to a class of equations that was discussed in [BH75] and in great detail by Ecalle in [Eca85, §3.6] and later by Immink in [Imm]. It has a unique formal solution $\hat{h}=\sum_{n \geq 1} h_{n} s^{-n}$. Let $\hat{u}(t):=\sum_{n \geq 1} \frac{h_{n}}{(n-1) !} t^{n-1}$, the formal Borel transform of $\hat{h}$. The power series $\hat{u}$ formally satisfies the convolution equation

$$
e^{-t} u(t)-a(1 * u)(t)=1
$$

This equation has the unique analytic solution

$$
u(t)=e^{t-a} e^{a e^{t}} .
$$

Thus $\hat{u}(t)$ coincides with the Taylor series at $t=0$ of this function and is actually a convergent power series which extends to a holomorphic function on C. The convergence of $\hat{u}$ implies that $\hat{h}$ is 1-Gevrey. By using Cauchy's formula for the coefficients in a convergent Taylor series, one may derive the more precise estimate

$$
\left|h_{n}\right| \leq K\left(\frac{n}{\log n}\right)^{n} A^{n}, \forall n \geq 2, \text { for some } K, A>0 .
$$

This type of estimate is typical of difference equations possessing a level $1^{+}$ (cf. [Imm88]).

The function $u(t)=e^{t-a} e^{a e^{t}}$ is bounded in the left half plane and, consequently, $\hat{h}(s)$ is 1 -summable in all directions in $\left(\frac{\pi}{2}, \frac{3 \pi}{2}\right)$. The 1 -sum $h_{l}(s)$ is analytic on the sector $0<\arg s<2 \pi$ and is a solution of (1.4).

In the right half plane $u(t)$ grows faster than exponentially of any order on the horizontal strips $\{t \in \mathbf{C} \mid \Re t>0$, $t \in(-\pi / 2, \pi / 2) \bmod 2 \pi\}$, but on the strips

$$
\{t \in \mathbf{C} \mid \Re t>0, \Im t \in(\pi / 2,3 \pi / 2) \bmod 2 \pi\}
$$

it decreases faster than exponentially of any order. Hence, the functions

$$
h_{r, n}(s):=\int_{C_{n}} e^{-s t} u(t) d t, n \in \mathbf{Z},
$$

with $C_{n}$ a path from 0 to $+\infty+i \theta, \theta \in(\pi / 2+2 n \pi, 3 \pi / 2+2 n \pi)$ (see Figure 1) are well defined and satisfy (1.4).

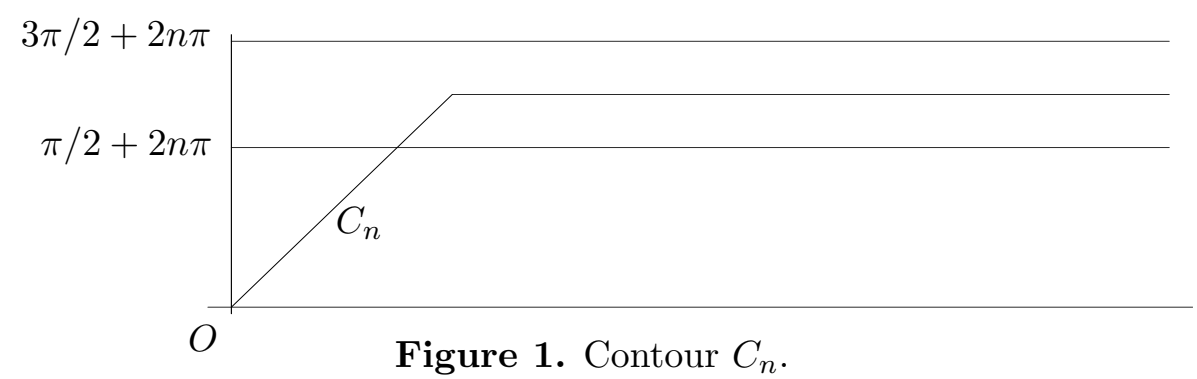


The functions $h_{r, n}$ all have the asymptotic expansion $\hat{h}$ as $s \rightarrow \infty,-\frac{\pi}{2}<$ $\arg s<\frac{\pi}{2}$, uniformly on closed subsectors (cf. also [BH75]). However, it can be shown that the $h_{r, n}$ are not 1-sums. See also [vdPS97, Chapter 11].

In order to characterize these solutions by means of their asymptotic behaviour, we have to consider this asymptotic behaviour on regions other than sectors, namely regions of the form

$$
D(\theta):=\{s \in \mathbf{C} \mid \Re\{s(\log s+i \theta)\}>1\}, \theta \in \mathbf{R},
$$

see Figures 2-5.

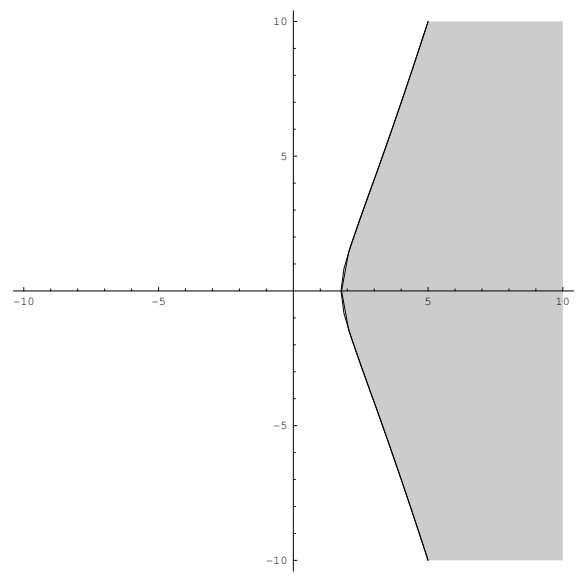

Figure 2. Region $D(0)$.

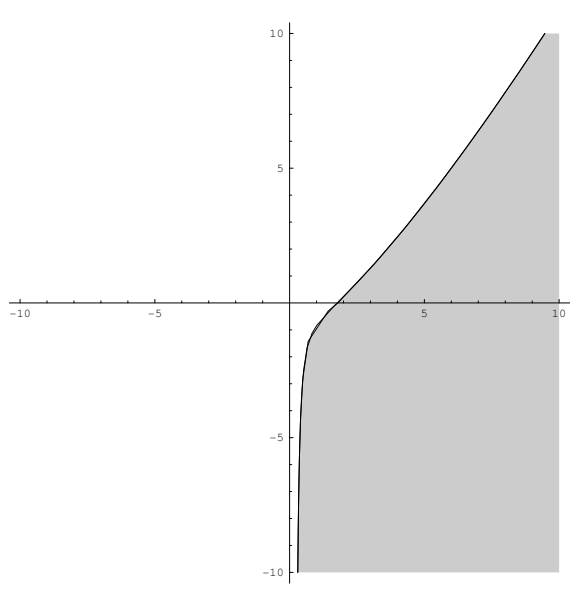

Figure 3. Region $D\left(\frac{\pi}{2}\right)$.

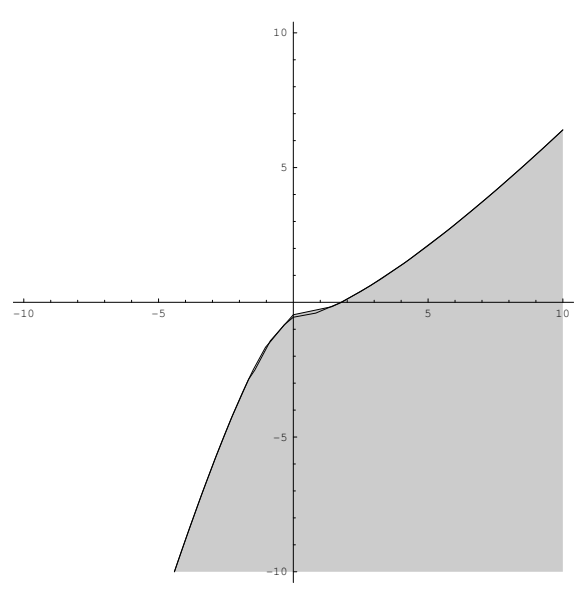

Figure 4. Region $D(\pi)$.

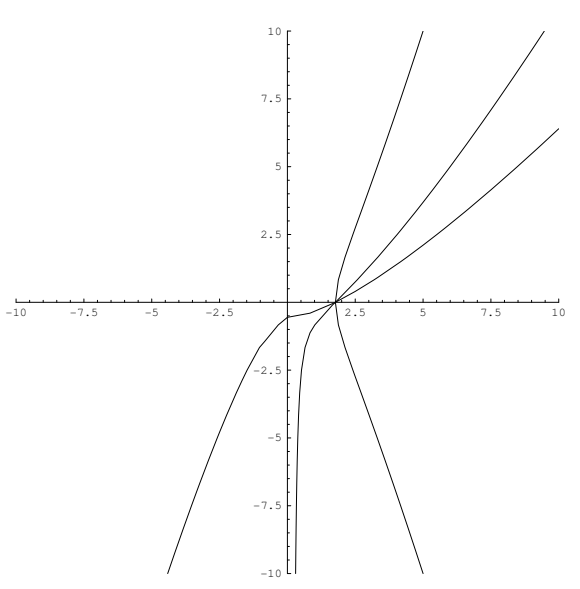

Figure 5. The regions 'rotate' clockwise with increasing $\theta$. 
Proposition. For any $\theta \in(2 n \pi, 2(n+1) \pi)$, there exist $K, A>0$ such that

$$
\left|h_{r, n}(s)-\sum_{n=1}^{N-1} h_{n} s^{-n}\right| \leq K A^{N}(N !)|s|^{-N}, \forall s \in D(\theta), \forall N \in \mathbf{N} .
$$

This proposition has been proved by Borel-Laplace methods in [Fab97]. According to a theorem by Immink in [Imm96], $h_{r, n}$ is uniquely determined by the above property.

\section{Preliminaries.}

By $\operatorname{Arg} z$ we denote the principal argument of $z \in \mathbf{C} \backslash\{0\}$; we take $\operatorname{Arg} z \in$ $(-\pi, \pi]$. The Riemann surface of the logarithm will be denoted by $\mathbf{C}_{\infty}$.

For $\alpha, \beta \in \mathbf{R}$ we denote by $S(\alpha, \beta)$ the open sector $\left\{s \in \mathbf{C}_{\infty} \mid \alpha<\arg s<\right.$ $\beta\}$, and by $S[\alpha, \beta]$ the closed sector $\left\{s \in \mathbf{C}_{\infty} \mid \alpha \leq \arg s \leq \beta\right\}$. Similarly, $S[\alpha, \beta)$ and $S(\alpha, \beta]$ denote half-open sectors. For $\mu \in \mathbf{Z}$ we define

$$
H_{\mu}:=S((\mu-1) \pi / 2,(\mu+1) \pi / 2) ; \bar{H}_{\mu}:=S[(\mu-1) \pi / 2,(\mu+1) \pi / 2] .
$$

Throughout this paper, by an upper half plane, a fourth quadrant, etcetera, we understand a lift of the upper half plane, the fourth quadrant, etcetera, from the complex plane to the Riemann surface of the logarithm. A sector will always be a sector of $\mathbf{C}_{\infty}$ with vertex at the origin.

By definition, a neighbourhood of $\infty$ in a sector $\mathbf{S}$ ( $S$ not necessarily open) is an open subset $U$ of $S$, such that, for any closed subsector $S^{\prime}$ of $S$ with aperture $\leq \pi$, we can find $s_{0} \in S$ such that $s_{0}+S^{\prime} \subset U$. In particular, $R e^{i \mu \pi / 2}+H_{\mu}, \mu \in \mathbf{Z}, R>0$, is a neighbourhood of $\infty$ both in $H_{\mu}$ and in $\bar{H}_{\mu}$.

If we write $f(s)=O(g(s))$ or $f(s)=o(g(s))$ as $s \rightarrow \infty$ on a sector $S$, we mean that $f$ and $g$ are functions defined on a neighbourhood $U$ of $\infty$ in $S$, and that the $O$ or $o$ relation holds uniformly, as $s \rightarrow \infty$, on the intersection of $U$ and any closed subsector of $S$.

Similarly, if $\hat{f}(s)=\sum_{j \geq 0} a_{j} s^{-j / p}$ where $p>0$, and if $S$ is a sector, then $f(s) \sim \hat{f}(s), s \rightarrow \infty$ on $S$, means the following: $f$ is an analytic function on a neighbourhood $U$ of $\infty$ in $S$ and for any closed subsector $S^{\prime} \subset S$ and any $N \in \mathbf{N}$, we can find positive constants $R$ and $C_{S^{\prime}, R, N}$, such that

$$
\left|f(s)-\sum_{j=0}^{N-1} a_{j} s^{-j / p}\right| \leq C_{S^{\prime}, R, N}|s|^{-N / p}, \forall s \in S^{\prime} \cap U,|s|>R .
$$

The set of such functions $f$ with an asymptotic expansion $\hat{f}$ on $S$ as above will be denoted by $\mathcal{A}(S)$. 
In accordance with the above, when we write $f(s) \sim 0, s \rightarrow \infty$ on $S$, we mean that $f(s)=o\left(s^{-N}\right), s \rightarrow \infty$ on $S$, for any $N \in \mathbf{N}$.

Suppose $\hat{u}(s)=\sum_{j=0}^{m} \hat{h}_{j}(s)(\log s)^{j}$ with $\hat{h}_{j} \in \mathbf{C}\left[\left[s^{-1 / p}\right]\right]$ for $j=0, \ldots, m$. If we write $u(s) \sim \hat{u}(s), s \rightarrow \infty$ on $S$, we mean that there exist analytic functions $h_{j}, j=0, \ldots, m$, on a neighborhood of $\infty$ in $S$ such that $u(s)=$ $\sum_{j=0}^{m} h_{j}(s)(\log s)^{j}$, with $h_{j}(s) \sim \hat{h}_{j}(s), s \rightarrow \infty$ on $S$.

If $f \in \mathcal{A}(S)$ such that (2.1) holds and if there exist $k>0$, and $K_{S^{\prime}, R}, A_{S^{\prime}, R}$ $>0$ such that, for each $N$,

$$
C_{S^{\prime}, R, N} \leq K_{S^{\prime}, R} A_{S^{\prime}, R}^{N} \Gamma\left(\frac{N}{p k}\right)
$$

then we call $f$ a $k$-Gevrey function on $S$ with respect to the family $\frac{1}{p} \mathbf{N}_{0}$, and we write $f \in \mathcal{A}_{(1 / k)}(S)$. Note that $\frac{1}{p} \mathbf{N}_{0}$ is an example of a 'convenient family', according to the terminology introduced by Malgrange in [Mal95].

Any $f \in \mathcal{A}_{(1 / k)}(S)$ has an asymptotic expansion $\hat{f}(s)=\sum_{j=0}^{\infty} a_{j} s^{-j / p}$ with the $a_{j}$ satisfying

$$
\left|a_{j}\right| \leq K A^{j} \Gamma\left(\frac{j}{p k}\right), \forall j>0,
$$

for some positive $K$ and $A$. Such a formal series $\hat{f}$ will be called a Gevrey series $\left(\right.$ in $s^{-1}$ ) of order $1 / k$ with respect to the family $\frac{1}{p} \mathbf{N}_{0}$, and $\mathbf{C}\left[\left[s^{-1 / p}\right]\right]_{\frac{1}{p k}}$ denotes the set of such series.

In the sequel all Gevrey functions and Gevrey series will be with respect to the family $\frac{1}{p} \mathbf{N}_{0}$ with $p$ as in (1.2), and we omit the references to this family in our notations.

A function $f$ defined on a neighbourhood of $\infty$ in a sector $S$ is exponentially small of order $k>0$ on $S$ if for any closed subsector $S^{\prime}$ of $S$ there exists a positive constant $c$ such that $f(s)=O\left(e^{-c|s|^{k}}\right), s \rightarrow \infty$ on $S^{\prime}$. If this holds for all positive $c$ then $f$ is said to be supra-exponentially small of order $k$ on $S$. The set of all analytic functions on a neighbourhood of $\infty$ in $S$ which are exponentially or supra-exponentially small of order $k$ on $S$ will be denoted by $\mathcal{A}^{\leq-k}(S)$ and $\mathcal{A}^{<-k}(S)$ respectively. If $S=S(\alpha, \beta)$ then we will also write these latter sets as $\mathcal{A}^{\leq-k}(\alpha, \beta)$ and $\mathcal{A}^{<-k}(\alpha, \beta)$. Similarly if $S=S(\alpha, \beta]$ etc. If $f$ and $g$ both are in $\mathcal{A}_{(1 / k)}(S)$, and $f$ and $g$ have the same asymptotic expansion, then it can be shown that their difference $f-g$ is in $\mathcal{A}^{\leq-k}(S)$ (cf. [Mal95]).

Let $l>0$ and $S$ be an open sector. Let $\left\{S_{i}\right\}_{i \in I}$ be a covering of $S$ consisting of open sectors and let $f^{(i)} \in \mathcal{A}\left(S_{i}\right), i \in I$, such that $f^{\left(i_{1}\right)}-f^{\left(i_{2}\right)} \in$ $\mathcal{A}^{\leq-l}\left(S_{i_{1}} \cap S_{i_{2}}\right)$ for any $i_{1}, i_{2} \in I$ with $S_{i_{1}} \cap S_{i_{2}} \neq \emptyset$. These data determine an $l$-precise quasi-function on $S$. We identify two such sets of data $\left(\left\{f^{(i)}\right\}_{i \in I} ;\left\{S_{i}\right\}_{i \in I}\right)$ and $\left(\left\{g^{(j)}\right\}_{j \in J} ;\left\{\tilde{S}_{j}\right\}_{j \in J}\right)$ if $f^{(i)}-g^{(j)} \in \mathcal{A}^{\leq-l}\left(S_{i} \cap \tilde{S}_{j}\right)$ where $i \in I, j \in J$ such that $S_{i} \cap \tilde{S}_{j} \neq \emptyset$. They define the same $l$-precise 
quasi-function on $S$ and we write $\left(\mathcal{A} / \mathcal{A}^{\leq-l}\right)(S)$ for the set of these $l$-precise quasi-functions on $S$. Indeed, if we identify the interval $I=(a, b)$ in $\mathbf{R}$ and the sector $S(a, b)$, then $\mathcal{A}$ and $\mathcal{A}^{\leq-k}, k>0$, can be considered as sheaves on $\mathbf{R}$ and then $\mathcal{A} / \mathcal{A}^{\leq-l}$ is the quotient sheaf. Also elements of $\left(\mathcal{A} / \mathcal{A}^{\leq-l}\right)^{n}(S)[\log s]$ with $n \in \mathbf{N}$ will be called $l$-precise quasi-functions on $S$.

All elements in a representative $\left\{f^{(i)}\right\}_{i \in I}$ of $f \in\left(\mathcal{A} / \mathcal{A}^{\leq-l}\right)^{n}(S)[\log s]$ have the same asymptotic expansion $\hat{f}$ and this expansion is independent of the chosen representative. Therefore we may write $f(s) \sim \hat{f}(s), s \rightarrow \infty$ on $S$, without causing confusion.

Similarly if $l>k>0$ we define $\left(\mathcal{A}_{(1 / k)} / \mathcal{A}^{\leq-l}\right)(S)$ as the set of $l$-precise quasi-functions $f$ which have representatives $f_{i}$ on $S_{i}$ as above with $f_{i} \in$ $\mathcal{A}_{(1 / k)}\left(S_{i}\right)$ for all $i \in I$.

If $\hat{f} \in \mathbf{C}\left[\left[s^{-1 / p}\right]\right]_{\frac{1}{p k}}$ then there exists a unique $f \in\left(\mathcal{A}_{(1 / k)} / \mathcal{A} \leq-k\right)\left(\mathbf{C}_{p}\right)$ such that $f \sim \hat{f}$, where $\mathbf{C}_{p}$ denotes the Riemann surface of $s^{1 / p}$. This $f$ will be denoted by $T^{-1} \hat{f}$ (cf. [MR92, Cor. (1.8)]).

Let $f \in\left(\mathcal{A} / \mathcal{A}^{\leq-l}\right)^{n}(S)[\log s]$. The 'restriction' of $f$ to an open subsector $S^{\prime}$ of $S$, denoted by $\left.f\right|_{S^{\prime}}$, is defined as follows: Suppose $\left\{f^{(i)}\right\}_{i \in I}$ is a representative of $f$ with respect to a covering $\left\{S_{i}\right\}_{i \in I}$ of $S$. Then $\left.f\right|_{S^{\prime}}$ is the element of $\left(\mathcal{A} / \mathcal{A}^{\leq-l}\right)^{n}\left(S^{\prime}\right)[\log s]$ defined by $\left\{\left.f^{(i)}\right|_{S_{i} \cap S^{\prime}}\right\}_{i \in I}$, where $\left.f^{(i)}\right|_{S_{i} \cap S^{\prime}}$ is the restriction of $f^{(i)}$ to a neighbourhood of $\infty$ in $S_{i} \cap S^{\prime}$.

Definition 2. Let $0<k_{1}<\cdots<k_{r}$, and let $\hat{f} \in \mathbf{C}\left[\left[s^{-1 / p}\right]\right]_{1 /\left(p k_{1}\right)}$. Furthermore, let $S_{1} \supset \ldots \supset S_{r}$ be a nested sequence of open sectors, where $S_{i}$ has aperture larger than $\pi / k_{i}, i=1, \ldots, r$ and $S_{1}$ has aperture at most $2 p \pi$. We say that $\hat{f}$ is $\left(k_{1}, \ldots, k_{r}\right)$-summable on $\left(S_{1}, \ldots, S_{r}\right)$ if there exist $f_{i} \in\left(\mathcal{A}_{\left(1 / k_{1}\right)} / \mathcal{A}^{\leq-k_{i+1}}\right)\left(S_{i}\right), i=1, \ldots, r-1$, and $f_{r} \in \mathcal{A}_{\left(1 / k_{1}\right)}\left(S_{r}\right)$, such that $\left.f_{i}\right|_{S_{i+1}} \equiv f_{i+1} \bmod \mathcal{A}^{\leq-k_{i+1}}, i=0, \ldots, r-1$ where $f_{0}=T^{-1} \hat{f}$. We call $f_{r}$ the $\left(k_{1}, \ldots, k_{r}\right)$-sum of $\hat{f}$ on $\left(S_{1}, \ldots, S_{r}\right)$, and we have $f_{r}(s) \sim \hat{f}(s), s \rightarrow \infty$ on $S_{r}$.

According to the 'relative Watson lemma' ([MR92, Prop. (2.1)]) $f_{i+1}$ is completely determined by $f_{i}$ and $S_{i+1}, i=0, \ldots, r-1$. Hence the $\left(k_{1}, \ldots, k_{r}\right)$-sum of $\hat{f}$ on $\left(S_{1}, \ldots, S_{r}\right)$ is uniquely defined. We may extend the definition of multisummability in an obvious way to the case that $\hat{f}$ is an $n$-vector or an $n \times n$-matix with elements in $\mathbf{C}\left[\left[s^{-1 / p}\right]\right]_{1 /\left(p k_{1}\right)}$.

Definition 3. Let $f(s)=s^{d s} e^{2 \pi i s b+q(s)} s^{\gamma}$, with $d \in \frac{1}{p} \mathbf{Z}, b \in \mathbf{C}, q(s)$ identically zero or a polynomial in $s^{1 / p}$ without constant term and of degree at most $p-1$, and $\gamma \in \mathbf{C}$. We will say that $f(s)$ is of level $1^{+}$if $d \neq 0$, of level 1 if $d=0, b \neq 0$, of level $k$ with $k \in\left\{\frac{1}{p}, \ldots, \frac{p-1}{p}\right\}$ if $d=b=0, q(s) \not \equiv 0$ and $q(s)$ has degree $p k$ in $s^{1 / p}$, and of level 0 if $d=b=0$ and $q(s) \equiv 0$. 
Let $f$ be of level $k \in(0,1]$, so $d=0,2 \pi i s b+q(s) \not \equiv 0$. A closed interval $[\sigma-\pi / k, \sigma]$ will be called a Stokes interval of level $k$ of $f$ if $f \in \mathcal{A}^{\leq-k}(\sigma-$ $\pi / k, \sigma)$. So if $k=1$ then $\sigma \equiv \pi-\operatorname{Arg} b \bmod 2 \pi$ whereas if $0<k<1$ and $q(s)=\omega s^{k}+o\left(s^{k}\right), s \rightarrow \infty, \omega \neq 0$, then $k \sigma \equiv \frac{3}{2} \pi-\operatorname{Arg} \omega \bmod 2 \pi$.

If $f$ is of level $1^{+}$we will, in Section 7 , associate with it a certain Stokes number. This number is connected with curves that separate regions of growth from regions of decay. All these curves have the limiting directions $\frac{\pi}{2} \bmod \pi$.

If $f$ is of level $1^{+}$, we have $f(s)=\exp (d s \log s(1+o(1))), s \rightarrow+\infty$ with $d \neq 0$, and so it grows or decays faster than exponentially of order 1 on $\mathbf{R}^{+}$, but slower than any higher exponential order.

Definition 4. For any (i.e., not necessarily open) sector $S$ we will write $f \in \mathcal{A}^{\leq-1^{+}}(S)$ to express that $f$ is analytic on a neighbourhood $U$ of $\infty$ in $S$, and that for any closed subsector $S^{\prime}$ of $S$, there exists a positive constant $c$ (depending on $S^{\prime}$ ) such that $f(s)=O\left(e^{-c|s| \log |s|}\right)$, uniformly as $s \rightarrow \infty$ on $S^{\prime} \cap U$.

We define $1^{+}$-precise quasi-functions by replacing $l$ by $1^{+}$in the definition of $l$-precise quasi-functions above.

So, for example, $e^{d s \log s} \in \mathcal{A}^{\leq-1^{+}}\left(H_{0}\right)$ if $d<0$. And if $p(s)=\sum_{j \geq 0} p_{j} e^{2 \pi i s j}$ is an analytic 1-periodic function on $\{s \in \mathbf{C} \mid \Im s>R\}$ for some $R>0$, then $p(s) e^{d s \log s} \in \mathcal{A}^{\leq-1^{+}}\left(H_{0} \cap \bar{H}_{1}\right)$ if $d<0$.

With Equation (1.1) we associate levels, and with each level certain Stokes intervals or numbers. For this purpose we rewrite the formal fundamental matrix solution (1.2) as follows:

$$
\hat{\mathrm{Y}}(s)=\hat{\mathrm{U}}(s) \mathrm{F}(s),
$$

where $\hat{\mathrm{U}}(s)=\hat{\mathrm{H}}(s) s^{\mathrm{N}}, \mathbf{N}=\bigoplus_{j=1}^{m} \mathrm{~N}_{j}$ and $\mathrm{F}(s)=s^{\Lambda s} e^{\mathrm{G}(s)} s^{\mathrm{C}}, \mathbf{C}=\bigoplus_{j=1}^{m} c_{j} \mathbf{l}_{j}$.

The columns $\hat{y}_{l}(s)(l=1, \ldots, n)$ of $\hat{Y}(s)$ form a formal fundamental system of solutions $\left\{\hat{y}_{l}\right\}_{l=1}^{n}$ and we have

$$
\hat{y}_{l}(s)=f_{l}(s) \hat{u}_{l}(s), f_{l}(s)=s^{d_{l} s} e^{2 \pi i s b_{l}+q_{l}(s)} s^{\gamma_{l}},
$$

where $\hat{u}_{l}(s) \in \mathbf{C}^{n}\left[\left[s^{-1 / p}\right]\right][\log s]$ is the $l$-th column of $\hat{\mathbf{U}}(s)$, and, furthermore, if $0<l-\left(n_{1}+\ldots+n_{j-1}\right) \leq n_{j}$, then $d_{l}=\lambda_{j}, b_{l} \in \mathbf{C}$ and $q_{l}(s) \equiv 0$ or $q_{l}(s)$ is a polynomial in $s^{1 / p}$ without constant term and of degree at most $p-1$ such that $2 \pi i s b_{l}+q_{l}(s)=g_{j}(s)$ and $\gamma_{l}=c_{j}$. Without loss of generality, we may assume that $\Re b_{l} \in[0,1), l=1, \ldots, n$.

We use the following abbreviations (cf. (2.3)): $f_{m l}:=f_{m} f_{l}^{-1}, d_{m l}:=$ $d_{m}-d_{l}, b_{m l}:=b_{m}-b_{l}, q_{m l}:=q_{m}-q_{l}, \gamma_{m l}:=\gamma_{m}-\gamma_{l}$. We write $\kappa_{m l}$ for the level of $f_{m l}$. 
Definition 5. The levels of Equation (1.1) are the levels of the functions $e^{2 \pi i s j} f_{m l}(s), j \in \mathbf{Z}, m, l \in\{1, \ldots, n\}$. Let $k \in\left\{\frac{1}{p}, \ldots, \frac{p-1}{p}, 1\right\}$. The Stokes intervals of level $k$ of the equation are the Stokes intervals of level $k$ of the functions $e^{2 \pi i s j} f_{m l}(s), j \in \mathbf{Z}, m, l \in\{1, \ldots, n\}$.

Taking $j=0$ we see that all the $\kappa_{m l}$ are levels of the equation. Moreover, 0 and 1 always are levels of the equation (take $m=l$ and then $j=0$ and $j \neq 0$, respectively). By $0<k_{1}<\cdots<k_{r}=1$ we denote the increasing sequence of levels of the equation in the interval $(0,1]$. If $\Im b_{m l} \neq 0$ for some $m$ and $l$ then there are infinitely many Stokes directions (endpoints of Stokes intervals) $-\operatorname{Arg}\left(b_{m l}+j\right) \bmod \pi, j \in \mathbf{Z}$ which cluster at $0 \bmod \pi$.

The following theorem is the counter part in the theory of linear difference equations of the 'main asymptotic existence' theorem in the theory of differential equations.

Theorem 6. Let $l \in\{1, \ldots, n\}$ and $\hat{y}_{l}(s)=f_{l}(s) \hat{u}_{l}(s)$ be a formal solution of (1.1) of the form (2.3) with $\hat{u}_{l}(s) \in \mathbf{C}^{n}\left[\left[s^{-1 / p}\right]\right][\log s]$.

Then for any $\mu \in \mathbf{Z}$ there exists an analytic solution $y_{l}(s)=f_{l}(s) u_{l}(s)$ of the equation such that $u_{l}(s) \sim \hat{u}_{l}(s), s \rightarrow \infty$ on $H_{\mu}$.

A proof of this theorem (for the case that no logarithmic terms appear in $\hat{u}(s))$ can be found in [vdPS97]. It is based on the so-called quadrant theorem, already stated by Birkhoff and Trjitzinsky in [BT33], but made rigorous by Immink in [Imm91].

\section{Two auxiliary lemmas.}

The following lemma gives information on the relation between two fundamental systems of solutions of Equation (1.1), which have the same asymptotic behaviour at $\infty$ on some sector.

Lemma 7. Suppose we have two fundamental matrix solutions of Equation (1.1), $\mathrm{Y}=\mathrm{UF}$ and $\mathrm{Y}_{1}=\mathrm{U}_{1} \mathrm{~F}$, such that $\mathrm{U}(s) \sim \hat{\mathrm{U}}(s)$ and $\mathrm{U}_{1}(s) \sim \hat{\mathrm{U}}(s)$ for $s \rightarrow \infty$ on an open sector $S$, with $\mathrm{F}(s)$ and $\hat{\mathrm{U}}(s)$ as in $(2.2)$. Let $u_{l}$ and $u_{l, 1}$ be the l-th column of $\mathrm{U}$ and $\mathrm{U}_{1}$, respectively.

Then there exist analytic 1-periodic functions $p_{l m}, m=1, \ldots, n$, on a neighbourhood of $\infty$ in $S$, such that

$$
u_{l}-u_{l, 1}=\sum_{m=1}^{n} p_{l m} f_{m l} u_{m} .
$$

Moreover,

$$
p_{l m}(s) f_{m l}(s) \sim 0, s \rightarrow \infty \text { on } S, \forall m \in\{1, \ldots, n\}
$$

If

$$
u_{l}-u_{l, 1} \in\left(\mathcal{A}^{\leq-k}\right)^{n}(S) \text { for some } k>0\left(\text { including } k=1^{+}\right),
$$


then

$$
p_{l m} f_{m l} \in \mathcal{A}^{\leq-k}(S), \forall m \in\{1, \ldots, n\} .
$$

Proof. Let $\widetilde{U}:=U-U_{1}$. Then $\widetilde{U} F=U F P$, or, equivalently, $F P F^{-1}=U^{-1} \widetilde{U}$, for some 1-periodic analytic matrix function $\mathrm{P}=\left(\mathrm{P}_{m l}\right)$, on a neighbourhood of $\infty$ in $S$. From the diagonal form of the matrix $\mathrm{F}$ it follows that if $p_{l m}$ is the element in the $m$-th row and $l$-th column of $\mathrm{P}$, then

$$
p_{l m} f_{m l}=\left(m \text {-th row of } \mathrm{U}^{-1}\right)\left(u_{l}-u_{l, 1}\right) .
$$

As $\mathrm{U}(s) \sim \hat{\mathrm{U}}(s)$ we have $\mathrm{U}^{-1}(s) \sim \hat{\mathrm{U}}^{-1}(s)=s^{-\mathrm{N}} \hat{\mathrm{H}}^{-1}(s)$. Hence any entry of $\mathrm{U}^{-1}$ is of order $O\left(s^{\mu}(\log s)^{\nu}\right), s \rightarrow \infty$ for some $\mu, \nu \in \mathbf{Z}$. Since $u_{l}(s)-$ $u_{l, 1}(s) \sim 0$ as $s \rightarrow \infty$ on $S$, we thus find that

$$
p_{l m}(s) f_{m l}(s) \sim 0, s \rightarrow \infty \text { on } S, \forall m \in\{1, \ldots, n\} .
$$

Similarly, we see that $p_{l m} f_{m l} \in \mathcal{A}^{\leq-k}(S)$, if $u_{l}-u_{l, 1} \in\left(\mathcal{A}^{\leq-k}\right)^{n}(S)$ for some $k>0$, including $k=1^{+}$.

The next lemma yields more information on the asymptotic behaviour of the functions $p_{l m} f_{m l}$ in the previous lemma.

Lemma 8. Let $S:=S\left(\alpha_{1}, \alpha_{2}\right)$ be an open sector with $0<\alpha_{2}-\alpha_{1} \leq \pi$. Let $p(s)$ be an analytic, 1-periodic function on a neighbourhood of $\infty$ in $S$, and let $f$ be a function of level $k \in\left\{0,1 / p, \ldots, 1,1^{+}\right\}$as in Section 2: $f(s)=s^{d s} e^{2 \pi i s b+q(s)} s^{\gamma}$ with $\Re b \in[0,1)$. Assume $g(s):=p(s) f(s) \sim 0$, $s \rightarrow \infty$ on $S$. Let $H$ be an upper or lower half plane in $\mathbf{C}_{\infty}$ which has a nonempty intersection with $S$.

Then $g \in \mathcal{A}^{\leq-k}(S)$ if $k>0$ and $g \in \mathcal{A}^{\leq-1}(H)$ if $k=0$. If $\nu$ denotes some integer we have:

1) If $\boldsymbol{k}=\mathbf{0}$ : If $\alpha_{1}<\nu \pi<\alpha_{2}$ then $p=g=0$.

2) If $\mathbf{0}<\boldsymbol{k}<\mathbf{1}$ :

Then there exists $c \in \mathbf{C}$ such that $g-c f \in \mathcal{A}^{\leq-1}(H)$ and $c f \in$ $\mathcal{A}^{\leq-k}(S)$. If $\alpha_{1}<\nu \pi<\alpha_{2}$ then $p(s)=c$. If $g \in \mathcal{A}^{<-k}(S)$ then $g \in \mathcal{A}^{\leq-1}(H)$.

3) If $\boldsymbol{k}=\mathbf{1}$ :

If $p \neq 0$ then with $H$ corresponds an integer $N$ such that $p(s) \sim$ $p_{N} e^{2 \pi i N s}$ as $|\Im s| \rightarrow \infty$ on $H$ where $p_{N} \neq 0$. If $b \in \mathbf{R}^{*}$ then $g \in$ $\mathcal{A}^{\leq-1}(H)$ and if moreover $\alpha_{1}<\nu \pi<\alpha_{2}$ then $p=g=0$. If $\alpha_{1} \leq$ $\nu \pi \leq \alpha_{2}$ and $(-1)^{\nu} \Im b<0$ then $p=g=0$.

Next suppose

(I) $\alpha_{1} \leq \nu \pi \leq \alpha_{2}$ and $(-1)^{\nu} \Im b>0$.

(II) $\left(\alpha_{1}, \alpha_{2}\right) \subset\left(\beta_{1}, \beta_{2}\right)$ where $\left(\beta_{1}, \beta_{2}\right)$ does not contain a Stokes interval of $e^{2 \pi i s j} f(s)$ of level 1 for any $j \in \mathbf{Z}$.

Then there exist analytic 1-periodic functions $p_{+}$and $p_{-}$such that $p=p_{+}+p_{-}$and $p_{+} f \in \mathcal{A}^{\leq-1}\left(\alpha_{1}, \beta_{2}\right)$ and $p_{-} f \in \mathcal{A}^{\leq-1}\left(\beta_{1}, \alpha_{2}\right)$. If 
$\alpha_{1}=\nu \pi$ then $p_{+} f \in \mathcal{A}^{\leq-1}\left[\nu \pi, \beta_{2}\right)$ and similarly if $\alpha_{2}=\nu \pi$ then $p_{-} f \in \mathcal{A}^{\leq-1}\left(\beta_{1}, \nu \pi\right]$.

4) If $\boldsymbol{k}=\mathbf{1}^{+}$:

If $\alpha_{1}<\left(\nu+\frac{1}{2}\right) \pi<\alpha_{2}$, then $p=g=0$. If $\nu \pi \leq \alpha_{1}<\alpha_{2} \leq$ $\left(\nu+\frac{1}{2}\right) \pi$ or $\left(\nu-\frac{1}{2}\right) \pi \leq \alpha_{1}<\alpha_{2} \leq \nu \pi$ then $g \in \mathcal{A}^{\leq-1^{+}}\left[\nu \pi, \alpha_{2}\right)$ and $g \in \mathcal{A}^{\leq-1^{+}}\left(\alpha_{1}, \nu \pi\right]$ respectively. If $f \notin \mathcal{A}^{\leq-1^{+}}(S)$ then $p=g=0$.

5) If $k \leq 1$ and $g \in \mathcal{A}^{<-1}(S)$ then $g=0$.

Proof. We will give the proof for the cases (i) $2 h \pi<\alpha_{1}<\alpha_{2}<(2 h+1) \pi$, (ii) $\alpha_{1}=2 h \pi$ and (iii) $\alpha_{1}<2 h \pi<\alpha_{2}$ for some $h \in \mathbf{Z}$. The other cases can be treated similarly.

We may choose $H=H_{4 h+1}$. So $p(s)$ and $g(s)$ are analytic on $H$ for $\Im s>R$ for some $R>0$. Put $z=e^{2 \pi i s}$ and $P(z):=p(s)$. Then $|z|=e^{-2 \pi \Im s}$ and we have expansions

$$
P(z)=\sum_{j=-\infty}^{\infty} p_{j} z^{j} \text { if } 0<|z|<e^{-2 \pi R}, p(s)=\sum_{j=-\infty}^{\infty} p_{j} e^{2 \pi i s j} \text { if } \Im s>R .
$$

In case (iii) $p$ is an entire function. So then (3.1) holds with $p_{j}=0$ if $j<0$ and $R$ may be replaced by $-\infty$. We now treat separately the different cases of the lemma.

Ad 1) We have $f(s)=s^{\gamma}$. Hence $p(s)=s^{-\gamma} g(s) \sim 0$ as $s \rightarrow \infty$ on $S$. The 1-periodicity of $p(s)$ then implies that $p(s) \sim 0$ as $\Im s \rightarrow \infty$, so $P(z) \rightarrow 0$ as $z \rightarrow 0$. Therefore $p_{j}=0$ if $j \leq 0$ and $p, g \in \mathcal{A}^{\leq-1}(H)$. In case (iii) also $p(s) \sim 0$ as $\Im s \rightarrow-\infty$, so $P(z) \rightarrow 0$ as $z \rightarrow \infty$. Hence $P=0$ and so $g=p=0$.

Ad 2) For any closed sector $S_{1} \subset(S \cap H)$ and any $\rho>R$, there exist positive constants $K$ and $a$ such that

$$
|p(s)|=\left|g(s) f(s)^{-1}\right| \leq K \exp \left(a|s|^{k}\right), \text { if } s \in S_{1} \text { and } \Im s \geq \rho .
$$

From the 1-periodicity of $p(s)$ and the fact that $k \in(0,1)$ it follows that $|p(s)| \leq K \exp \left(a^{\prime} \Im s\right), \forall \Im s \geq \rho$, for some $a^{\prime} \in(0,2 \pi)$, if we choose $\rho$ sufficiently large. This implies $P(z)=o\left(z^{-1}\right), z \rightarrow 0$. Hence $p_{j}=0$ if $j<0$ in (3.1). With $c:=p_{0}$ we get $p(s)-c \in \mathcal{A}^{\leq-1}(H)$ and $g-c f \in \mathcal{A}^{\leq-1}(H)$. If $f \notin \mathcal{A} \leq-k(S \cap H)$ then $c=0$ since otherwise $g$ is unbounded in a neighbourhood of $\infty$ in $S$. So in cases (i) and (ii) we have $c f \in \mathcal{A}^{\leq-k}(S)$. Also $c=0$ if $g \in \mathcal{A}^{<-k}(S)$ and therefore $g \in \mathcal{A}^{\leq-1}(H)$. In case (iii) we have moreover $P(z)=o(z)$ as $z \rightarrow \infty$ and so $P(z)=p(s) \equiv c$. If $c \neq 0$ then $g=c f \in \mathcal{A}^{\leq-k}(S)$ as $g \sim 0$ in $S$.

Ad 3) The fact that $f$ is of level 1 implies that $d=0, b \neq 0$.

We have $p(s)=g(s) f(s)^{-1}=O(1) \exp (-2 \pi i s(b+o(1)))$ as $s \rightarrow \infty$ on $S \cap H$ and therefore $P(z)=O\left(z^{b+o(1)}\right)$ as $z \rightarrow 0$. So if $p \neq 0$ then there exists $N \in \mathbf{Z}$ such that $p_{j}=0$ for all $j<N$ and $p_{N} \neq 0$ in (3.1). Then 
$p(s)=p_{N} e^{2 \pi i N s}(1+o(1))$ and $g(s)=p_{N} e^{2 \pi i(b+N+o(1)) s}(1+o(1))$ as $\Im s \rightarrow \infty$. As $g(s) \sim 0$ as $s \rightarrow \infty$ in $S$ it follows that $g \in \mathcal{A}^{\leq-1}(S \cap H)$ where $S \cap H=S$ in cases (i) and (ii). Moreover, if $\Im b=0$ then $b+N>0$ and so $g \in \mathcal{A}^{\leq-1}(H)$. In cases (ii) and (iii) we see that if $p \neq 0$ and $\Im b<0$ then $g(s) \rightarrow \infty$ on $\arg s=2 h \pi+\epsilon$ for $\epsilon$ sufficiently small positive. Hence $p=g=0$ if $\Im b<0$.

In case (iii) similar reasoning as above leads to $p_{j}=0$ for all $j>M$ with some $M \in \mathbf{Z}, M \geq N, g(s)=e^{2 \pi i(b+M+o(1)) s}\left(p_{M}+o(1)\right)$ as $\Im s \rightarrow-\infty$ and $g \in \mathcal{A}^{\leq-1}\left(\alpha_{1}, 2 h \pi\right)$. In particular, if $\Im b=0$ and $p_{N} \neq 0 \neq p_{M}$, then the fact that $g \sim 0$ in $S(2 h \pi-\epsilon, 2 h \pi+\epsilon)$ for some $\epsilon>0$ implies that $N+b>0>M+b$ in contradiction with $M \geq N$, and therefore $p=g=0$. So in case (iii) we have $\Im b \neq 0$ if $p \neq 0$ and so $\Im b>0$. Consequently $g$ is exponentially small of order 1 in $S(2 h \pi-\epsilon, 2 h \pi+\epsilon)$ for some $\epsilon>0$. Thus $g \in \mathcal{A}^{\leq-1}(S)$.

Next consider the case that (I) and (II) are satisfied. Now $\nu=2 h$ and only cases (ii) and (iii) with $\Im b>0$ have to be considered. Let $\sigma_{j}:=$ $(2 h+1) \pi-\operatorname{Arg}(b+j)$ for all $j \in \mathbf{Z}$. Then $S\left(\sigma_{j}-\pi, \sigma_{j}\right)$ is a maximal sector where $e^{2 \pi i s j} f(s)$ is exponentially small of order 1 and the behaviour of $g$ on $S \cap H$ implies that $\sigma_{N}-\pi \leq 2 h \pi<\alpha_{2} \leq \sigma_{N}$. If $\sigma_{N} \geq \beta_{2}$ then we see that $g \in \mathcal{A}^{\leq-1}\left[2 h \pi, \beta_{2}\right)$. Next suppose $\sigma_{N}<\beta_{2}$. As $\sigma_{j}$ increases monotonically from $2 h \pi$ to $(2 h+1) \pi$ as $j$ increases from $-\infty$ to $+\infty$, there exists $A \in \mathbf{Z}$ such that $\sigma_{A}<\beta_{2} \leq \sigma_{A+1}$ and $A \geq N$. The condition on Stokes intervals now implies that $\sigma_{A}-\pi \leq \beta_{1}$.

Let $p_{-}(s):=\sum_{j=N}^{A} p_{j} e^{2 \pi i s j}$. Then $p_{-}(s)=O\left(e^{2 \pi i s A}\right)$ on the lower half plane and $p_{-}(s)=O\left(e^{2 \pi i s N}\right)$ on the upper half plane. So $p_{-} f$ is exponentially small of order 1 for $\arg s \in\left(\sigma_{A}-\pi, 2 h \pi\right]$ and for $\arg s \in$ $\left[2 h \pi, \sigma_{N}\right)$. Hence $p_{-} f \in \mathcal{A}^{\leq-1}\left(\beta_{1}, \alpha_{2}\right)$. Furthermore, $p_{+}:=p-p_{-}=$ $\sum_{j=A+1}^{\infty} p_{j} e^{2 \pi i s j}=O\left(e^{2 \pi i s(A+1)}\right)$ on the upper half plane and as $\sigma_{A+1} \geq \beta_{2}$ we see that $p_{+} f \in \mathcal{A}^{\leq-1}\left[2 h \pi, \beta_{2}\right)$. Moreover, $p_{+} f=g-p_{-} f \in \mathcal{A}^{\leq-1}\left(\alpha_{1}, \alpha_{2}\right)$ and we conclude that $p_{+} f \in \mathcal{A}^{\leq-1}\left(\alpha_{1}, \beta_{2}\right)$.

Ad 4) Now $f(s)=\exp \{d s(\log s+O(1))\}, s \rightarrow \infty$ with $d \neq 0$. Since $\Re(s \log s)=\Re s \log |s|-\Im s \arg s$, we have $p(s)=g(s) f(s)^{-1}=$ $O(\exp \{-d \Re s \log |s|+O(s)\})$, as $s \rightarrow \infty$ on $S$. As $\Re s / \Im s$ is a nonzero constant on any ray $\arg s=\psi \notin \frac{\pi}{2} \mathbf{Z}$ we see that if $S$ contains such a ray on which $d \Re s>0$ then $p(s)=O(1) \exp (-N|\Im s|)$ for any $N \in \mathbf{N}$ and therefore $p=g=0$. In particular, if $f \notin \mathcal{A}^{\leq-1^{+}}(S)$ then there exists a ray where $d \Re s>0$, so $p=g=0$.

It is now sufficient to consider the case that $S$ belongs to a right half plane and $d<0$. Choose $\varepsilon$ with $0<\varepsilon<\left(\alpha_{2}-\alpha_{1}\right) / 2$ and $\varepsilon<\alpha_{2} / 2-h \pi$. Let $\psi \in\left(2 h \pi, \alpha_{2}-2 \varepsilon\right)$. For any $s$ with $\arg s=\psi$ there exists $s_{-} \in H$ with $s-s_{-} \in \mathbf{N}$ and $\arg s_{-} \in\left(\alpha_{2}-\varepsilon, \alpha_{2}-\varepsilon / 2\right)$ if $\Im s$ is sufficiently large. Then $\Re s_{-}<\Im s \cot \left(\alpha_{2}-\varepsilon\right)$. Thus

$$
p(s)=p\left(s_{-}\right)=O(1) \exp \left[-d \cot \left(\alpha_{2}-\varepsilon\right) \Im s \log \Im s+O(s)\right] .
$$


From this and $\Im s \log \Im s=|s| \sin \psi \log (|s| \sin \psi)=|s|(\sin \psi \log |s|+O(1))$ we conclude that $|p(s)| \leq K_{1} \exp \left\{-d \cot \left(\alpha_{2}-\varepsilon\right) \sin \psi|s| \log |s|+K_{2}|s|\right\}$ if $\Im s$ is sufficiently large where $K_{1}$ and $K_{2}$ are some positive constants. Using $g(s)=O(1) p(s) \exp [-|d s|(\cos \psi) \log |s|+O(s)]$ and $\cos \psi-(\sin \psi) \cot \left(\alpha_{2}-\right.$ $\varepsilon)=\sin \left(\alpha_{2}-\varepsilon-\psi\right) / \sin \left(\alpha_{2}-\varepsilon\right)>\sin \varepsilon / \sin \left(\alpha_{2}-\varepsilon\right)=: c_{\varepsilon}>0$ we see that

$$
g(s)=O(1) \exp \left(-c_{\varepsilon}|d s| \log |s|\right)
$$

if $\Im s$ is sufficiently large and $\arg s \in\left(2 h \pi, \alpha_{2}-2 \varepsilon\right)$. Thus we see that $g \in \mathcal{A}^{\leq-1^{+}}\left[2 h \pi, \alpha_{2}\right)$. In case (iii) we get similarly $g \in \mathcal{A}^{\leq-1^{+}}\left(\alpha_{1}, 2 h \pi\right]$. Furthermore, the 1-periodic function $p$ is bounded on any bounded strip parallel to the real axis intersected with $S$. Thus we obtain $g \in \mathcal{A}^{\leq-1^{+}}(S)$ in case (iii).

Ad 5) If $g(s) \in \mathcal{A}^{<-1}(S)$ and $k \leq 1$ we deduce $p(s)=g(s) / f(s) \in$ $\mathcal{A}^{<-1}(S)$. Therefore $p(s)=O(1) e^{-c|s|}$ as $s \rightarrow \infty$ for all $c>0$. So $P(z)=$ $O\left(z^{j}\right)$ as $z \rightarrow 0$ for all $j$. Hence $P(z) \equiv 0$ and so $p=g=0$.

\section{A Gevrey property of solutions}

Proposition 9. Let $k_{1}$ be the lowest positive level of (1.1). Then the elements of $\hat{\mathrm{H}}(s)$ are Gevrey series of order $1 / k_{1}$. There exist fundamental matrices $\mathrm{Y}^{(\mu)}(s)=\mathrm{H}^{(\mu)}(s) s^{\Lambda s} e^{\mathrm{G}(s)} s^{\mathrm{L}}$ of (1.1) such that $\mathrm{H}^{(\mu)}(s)$ is a matrix of $k_{1}$-Gevrey functions on $H_{\mu}$ with $\mathrm{H}^{(\mu)}(s) \sim \hat{\mathrm{H}}(s)$ on $H_{\mu}$ for all $\mu \in \mathbf{Z}$. For any $\mu_{0} \in \mathbf{Z}$ a representative of $T^{-1} \hat{\mathbf{H}}$ (cf. definition of $T^{-1}$ in Section 2) on the covering $\left\{H_{\mu} \mid \mu=\mu_{0}, \ldots, \mu_{0}+4 p-1\right\}$ of $\mathbf{C}_{p}$ is given by $\left\{\mathrm{H}^{(\mu)} \mid \mu=\mu_{0}, \ldots, \mu_{0}+4 p-1\right\}$.

Let $S$ be an open sector of aperture at most $\pi$ and let $\hat{u}_{l}$ be given by (2.3) for $l=1, \ldots, n$. Assume that $f_{l} v_{l}$ is a solution of (1.1) such that $v_{l} \sim \hat{u}_{l}$ on $S$ for $l=1, \ldots, n$. Then $v_{l} \in\left(\mathcal{A}_{\left(1 / k_{1}\right)}\right)^{n}(S)[\log s]$. Moreover, $\left\{f_{l} v_{l}\right\}_{l=1}^{n}$ is a fundamental set of solutions of equation (1.1).

Proof. To prove the last statement, let $\mathrm{V}$ be the matrix with $v_{l}$ as $l$-th column. Then $\mathrm{V} \sim \hat{\mathrm{U}}$ on $S$, where $\hat{\mathrm{U}}$ as in (2.2). As $\operatorname{det} \hat{\mathrm{U}} \not \equiv 0$, we also have $\operatorname{det} \mathrm{V} \not \equiv 0$. Thus $\mathrm{Y}:=\mathrm{VF}$ is a matrix solution of Equation (1.1) and $\operatorname{det} Y \not \equiv 0$, i.e., it is a fundamental matrix solution.

According to Theorem 6 and the last statement of the proposition under consideration we have fundamental matrices $\mathrm{Y}^{(\mu)}(s)=\mathrm{H}^{(\mu)}(s) s^{\mathrm{N}} \mathrm{F}(s), \mu=$ $\mu_{0}, \ldots, \mu_{0}+4 p-1$, with $\mathrm{H}^{(\mu)}(s) \sim \hat{\mathrm{H}}(s), s \rightarrow \infty$ on $H_{\mu}, \hat{\mathrm{H}}(s)$ as in (1.2). Since $e^{2 p \pi i} H_{\mu}=H_{\mu+4 p}$ we define $\mathbf{H}^{\left(\mu_{0}+4 p\right)}(s)=\mathrm{H}^{\left(\mu_{0}\right)}\left(s e^{-2 p \pi i}\right), s \in H_{\mu_{0}+4 p}$. Then $\mathrm{H}^{\left(\mu_{0}+4 p\right)}(s) \sim \hat{\mathrm{H}}\left(s e^{-2 p \pi i}\right)=\hat{\mathrm{H}}(s), s \rightarrow \infty$ on $H_{\mu_{0}+4 p}$. If $s \in H_{\mu_{0}+4 p}$, $\zeta:=s e^{-2 p \pi i} \in H_{\mu_{0}}$, then $s+1=(\zeta+1) e^{2 p \pi i}$ and

$$
\begin{aligned}
\mathrm{Y}^{\left(\mu_{0}+4 p\right)}(s) & :=\mathrm{H}^{\left(\mu_{0}+4 p\right)}(s) s^{\mathrm{N}} \mathrm{F}(s) \\
& =\mathrm{H}^{\left(\mu_{0}\right)}(\zeta)\left(\zeta e^{2 p \pi i}\right)^{\mathrm{N}} \mathrm{F}\left(\zeta e^{2 p \pi i}\right)=\mathrm{Y}^{\left(\mu_{0}\right)}(\zeta) \mathrm{P}(\zeta),
\end{aligned}
$$


where $\mathrm{P}(\zeta)=e^{2 p \pi i(\zeta \Lambda+\mathrm{L})}$, with $\Lambda$ and $\mathrm{L}$ as in (1.2), is a 1-periodic matrix function, and $\operatorname{det} P(\zeta) \neq 0$. Hence $\mathrm{Y}^{\left(\mu_{0}+4 p\right)}(s)$ is a fundamental matrix.

Next we prove that the entries of $\mathrm{H}^{(\mu)}(s)$ are in $\mathcal{A}_{\left(1 / k_{1}\right)}\left(H_{\mu}\right), \mu=\mu_{0}, \ldots$, $\mu_{0}+4 p$. As the half planes $H_{\mu}, \mu=\mu_{0}, \ldots, \mu_{0}+4 p-1$, cover a neighbourhood of $\infty$ on the Riemann surface of $z^{1 / p}$, it is, by [MR92, Theorem 1.6], sufficient to prove that the entries of $\mathrm{H}^{(\mu+1)}(s)-\mathrm{H}^{(\mu)}(s)$ are exponentially small of order $k_{1}$ on $H_{\mu} \cap H_{\mu+1}, \mu=\mu_{0}, \ldots, \mu_{0}+4 p-1$. If we denote by $u_{l}^{(\mu)}(s)$ the $l$-th column of $\mathrm{H}^{(\mu)}(s) s^{\mathrm{N}}, l=1, \ldots, n, \mu=\mu_{0}, \ldots, \mu_{0}+4 p$, this is equivalent to proving that the differences $u_{l}^{(\mu+1)}-u_{l}^{(\mu)}$ are in $\left(\mathcal{A}^{\leq-k_{1}}\right)^{n}\left(H_{\mu} \cap H_{\mu+1}\right)$. We have

$$
u_{l}^{(\mu+1)}-u_{l}^{(\mu)}=\sum_{m=1}^{n} p_{l m} f_{m l} u_{m}^{(\mu)},
$$

for some 1-periodic functions $p_{l m}$ on a neighbourhood of $\infty$ in $H_{\mu} \cap H_{\mu+1}$. Since $u_{l}^{(\mu+1)}(s)-u_{l}^{(\mu)}(s) \sim 0$, as $s \rightarrow \infty$ on $H_{\mu} \cap H_{\mu+1}$, we have $p_{l m}(s) f_{m l}(s) \sim$ $0, s \rightarrow \infty$ on $H_{\mu} \cap H_{\mu+1}$, for $m=1, \ldots, n$, according to Lemma 7 . Lemma 8 now yields that $p_{l m} f_{m l} \in \mathcal{A}^{\leq-k_{1}}\left(H_{\mu} \cap H_{\mu+1}\right), m=1, \ldots, n$, so

$$
u_{l}^{(\mu+1)}-u_{l}^{(\mu)} \in\left(\mathcal{A}^{\leq-k_{1}}\right)^{n}\left(H_{\mu} \cap H_{\mu+1}\right) .
$$

Applying [MR92, Theorem 1.6], we conclude that

$$
u_{l}^{(\mu)} \in\left(\mathcal{A}_{\left(1 / k_{1}\right)}\right)^{n}\left(H_{\mu}\right)[\log s], l=1, \ldots, n, \mu=\mu_{0}, \ldots, \mu_{0}+4 p-1,
$$

$\mathrm{H}^{(\mu)}$ is a $k_{1}$-Gevrey function on $H_{\mu}$ and the elements of $\hat{\mathrm{H}}(s)$ are Gevrey series of order $1 / k_{1}$. Moreover, it follows that $\left\{\mathrm{H}^{(\mu)} \mid \mu=\mu_{0}, \ldots, \mu_{0}+4 p-1\right\}$ is a representative of $T^{-1} \hat{\mathrm{H}}$.

Finally we prove the statement concerning the functions $v_{l}(s)$. It is sufficient to consider the case that $S \subset\left(H_{\mu_{0}} \cup H_{\mu_{0}+1}\right)$. There exist 1-periodic functions $\tilde{p}_{l m}$ analytic on a neighbourhood of $\infty$ in $S \cap H_{\mu_{0}}$, such that

$$
v_{l}-u_{l}^{\left(\mu_{0}\right)}=\sum_{m=1}^{n} \tilde{p}_{l m} f_{m l} u_{m}^{\left(\mu_{0}\right)} .
$$

Since $v_{l}(s)-u_{l}^{\left(\mu_{0}\right)}(s) \sim 0$, as $s \rightarrow \infty$ on $S \cap H_{\mu_{0}}$, Lemma 7 and Lemma 8 now tell us that $\tilde{p}_{l m} f_{m l} \in \mathcal{A}^{\leq-k_{1}}\left(S \cap H_{\mu_{0}}\right)$, hence

$$
v_{l}-u_{l}^{\left(\mu_{0}\right)} \in\left(\mathcal{A}^{\leq-k_{1}}\right)^{n}\left(S \cap H_{\mu_{0}}\right) .
$$

The same holds with $\mu_{0}$ replaced by $\mu_{0}+1$. It follows that $v_{l} \in$ $\left(\mathcal{A}_{\left(1 / k_{1}\right)}\right)^{n}(S)[\log s]$, what had to be proven. 


\section{Refinement of chains of solutions.}

Consider the fundamental matrix $\mathrm{Y}^{(\mu)}(s)$ of Proposition 9 for $\mu \in \mathbf{Z}$. Let its columns be denoted by $\left\{f_{l} u_{l, 0}^{(\mu)}\right\}$ for $l=1, \ldots, n$ as in (2.3). Then $u_{l, 0}^{(\mu)} \in$ $\left(\mathcal{A}_{\left(1 / k_{1}\right)}\right)^{n}\left(H_{\mu}\right)[\log s], u_{l, 0}^{(\mu)} \sim \hat{u}_{l}$ on $H_{\mu}$ and $\left\{u_{l, 0}^{(\mu)} \mid \mu=\mu_{0}, \ldots, \mu_{0}+4 p-\right.$ 1 ) represents the $k_{1}$-precise quasi-function corresponding to $T^{-1} \hat{u}_{l}$ on the covering $\left\{H_{\mu} \mid \mu=\mu_{0}, \ldots, \mu_{0}+4 p-1\right\}$ of $\mathbf{C}_{p}$ for any $\mu_{0} \in \mathbf{Z}$.

In this section we show how these $k_{1}$-precise quasi-functions can be refined to $k_{2}$-precise quasi-functions with representatives $\left\{u_{l, 1}^{(\mu)}\right\}$ on an open sector $S\left(\alpha_{1}, \beta_{1}\right)$ with aperture $>\pi / k_{1}$ such that $\left(\alpha_{1}, \beta_{1}\right)$ does not contain a Stokes interval of level $k_{1}$ of the equation and such that $\left\{f_{l} u_{l, 1}^{(\mu)}\right\}_{l=1}^{n}$ again is a fundamental system of (1.1) with the same asymptotic expansion as before. This will be done by expressing the differences $f_{l}\left(u_{l, 0}^{(\mu)}-u_{l, 0}^{(\mu+1)}\right)$ in terms of suitable fundamental systems and distributing the terms that are $f_{l}$ times an exponentially small factor of order $k_{1}$, over $u_{l, 0}^{(\mu)}$ and $u_{l, 0}^{(\mu+1)}$. The same method can be applied to proceed from $k_{j}$-precise quasi-functions $u_{l, j-1}^{(\mu)}$ to $k_{j+1}$-precise quasi-functions $u_{l, j}^{(\mu)}$ corresponding to solutions $f_{l} u_{l, j-1}^{(\mu)}$ and $f_{l} u_{l, j}^{(\mu)}$ of $(1.1)$.

Proposition 10. Let $0<k_{1}<\cdots<k_{r}=1$ be the levels in $(0,1]$ of (1.1). Let $j \in\{1, \ldots, r\}$, and define $k:=k_{j}$. If $j<r$, then $k^{\prime}:=k_{j+1}$, otherwise $k^{\prime}:=1^{+}$. Let $(\alpha, \beta)$ be an open interval of length $>\pi / k$ not containing a Stokes interval of level $k$ of (1.1). Let $M$ and $N$ be the integers, such that $(M-1) \frac{\pi}{2} \leq \alpha<M \frac{\pi}{2}<N \frac{\pi}{2}<\beta \leq(N+1) \frac{\pi}{2}$. Define $\Gamma_{\mu}:=H_{\mu} \cap S(\alpha, \beta)$ for $\mu=M, \ldots, N$.

Suppose that we have fundamental systems of solutions $\left\{f_{l} u_{l}^{(\mu)}\right\}_{l=1}^{n}$ on $\Gamma_{\mu}$ for $\mu=M, \ldots, N$ which satisfy for $l=1, \ldots, n$ :

(i) $u_{l}^{(\mu)} \in\left(\mathcal{A}_{\left(1 / k_{1}\right)}\right)^{n}\left(\Gamma_{\mu}\right)[\log s], u_{l}^{(\mu)}(s) \sim \hat{u}_{l}(s), s \rightarrow \infty$ on $\Gamma_{\mu}$,

(ii) $u_{l}^{(\mu+1)}-u_{l}^{(\mu)} \in\left(\mathcal{A}^{\leq-k}\right)^{n}\left(H_{\mu} \cap H_{\mu+1}\right)$.

Then there exist fundamental systems of solutions $\left\{f_{l} \tilde{u}_{l}^{(\mu)}\right\}_{l=1}^{n}$ for $\mu=$ $M, \ldots, N$ such that for $l=1, \ldots, n$ :

$$
\tilde{u}_{l}^{(\mu)}-u_{l}^{(\mu)} \in\left(\mathcal{A}^{\leq-k}\right)^{n}\left(\Gamma_{\mu}\right) \text {, if } \mu \in\{M, \ldots, N\}
$$

and

$$
\tilde{u}_{l}^{(\mu+1)}-\tilde{u}_{l}^{(\mu)} \in\left(\mathcal{A}^{\leq-k^{\prime}}\right)^{n}\left(H_{\mu} \cap H_{\mu+1}\right) \text {, if } \mu \in\{M, \ldots, N-1\} .
$$

Moreover, for each $l \in\{1, \ldots, n\}$ the family of functions $\left\{\tilde{u}_{l}^{(\mu)}\right\}_{\mu=M}^{N}$ defines an element $\tilde{u}_{l}$ in $\left(\mathcal{A}_{\left(1 / k_{1}\right)} / \mathcal{A}^{\leq-k^{\prime}}\right)^{n}(\alpha, \beta)[\log s]$, which is uniquely determined by the properties of the $\tilde{u}_{l}^{(\mu)}$ mentioned above. 
We prove the proposition subsequently for the cases $k \in(0,1)$ and $k=1$. Proof for $\mathbf{k} \in(0,1)$.

We introduce the following sets:

$\mathrm{St}^{-}(\mu, l)=\left\{m \mid \kappa_{m l}=k, f_{m l} \in \mathcal{A}^{\leq-k}(\alpha,(\mu+1) \pi / 2)\right\}$ if $\mu \in\{M-1, \ldots, N-$ $1\}$;

$\mathrm{St}^{+}(\mu, l)=\left\{m \mid \kappa_{m l}=k, f_{m l} \in \mathcal{A}^{\leq-k}(\mu \pi / 2, \beta)\right\}$ if $\mu \in\{M, \ldots, N\}$. Obviously

$$
\mathrm{St}^{-}(\mu+1, l) \subset \mathrm{St}^{-}(\mu, l) \text {, and } \mathrm{St}^{+}(\mu-1, l) \subset \mathrm{St}^{+}(\mu, l) .
$$

Because of the assumption that $\beta-\alpha>\pi / k$ the two sets $\operatorname{St}^{-}(\mu, l)$ and $\mathrm{St}^{+}(\mu, l)$ are disjoint. Since $f_{j l}=f_{j m} f_{m l}$ the following transitivity relation holds:

$$
j \in \mathrm{St}^{-}(\mu, m) \wedge m \in \mathrm{St}^{-}(\mu, l) \Rightarrow j \in \mathrm{St}^{-}(\mu, l) .
$$

Finally, let $\mathrm{St}(\mu, l):=\mathrm{St}^{-}(\mu, l) \cup \mathrm{St}^{+}(\mu, l), \mu=M, \ldots, N-1$. If $\mu \in$ $\{M, \ldots, N-1\}$ then

$$
\kappa_{m l}=k \text { and } f_{m l} \in \mathcal{A}^{\leq-k}\left(H_{\mu} \cap H_{\mu+1}\right) \Longleftrightarrow m \in \operatorname{St}(\mu, l) .
$$

We only give the proof that the left statement implies the right one since the converse is trivial. The left-hand side implies that $f_{m l}$ has a Stokes interval $[\sigma-\pi / k, \sigma]$ containing $(\mu \pi / 2,(\mu+1) \pi / 2)$. Because of the assumptions of the proposition we have either $\alpha<\sigma-\pi / k<\beta \leq \sigma$ or $\sigma-\pi / k \leq \alpha<\sigma<\beta$ and therefore $m \in \operatorname{St}(\mu, l)$.

For $k<1$ the first statement of Proposition 10 is an easy consequence of the following two lemmas.

Lemma 11. Under the assumptions of Proposition 10 with $k<1$ there exist fundamental systems $\left\{f_{l} u_{l, 1}^{(\mu)}\right\}_{l=1}^{n}, \mu=M, \ldots, N$, satisfying:

(i) $u_{l, 1}^{(\mu)}-u_{l}^{(\mu)} \in\left(\mathcal{A}^{\leq-k}\right)^{n}\left(\Gamma_{\mu}\right)$;

(ii) $u_{l, 1}^{(\mu+1)}-u_{l, 1}^{(\mu)}=\sum_{m \in S t^{-}(\mu, l)} c_{m}^{(\mu)} f_{m l} u_{m, 1}^{(\mu)}+\psi_{l, 1}^{(\mu)}$, where $c_{m}^{(\mu)} \in \mathbf{C}$ and $\psi_{l, 1}^{(\mu)} \in\left(\mathcal{A}^{\leq-k^{\prime}}\right)^{n}\left(H_{\mu} \cap H_{\mu+1}\right)$.

Proof. The proof goes by induction on $\mu$. Define $u_{m, 1}^{(M)}:=u_{m}^{(M)}, m=$ $1, \ldots, n$. Next assume $u_{m, 1}^{(M)}, \ldots, u_{m, 1}^{(\mu)}$ have been defined for all $m=1, \ldots, n$ and some $\mu \in\{M, \ldots, N-1\}$. In the remaining part of this section $m$ will always be understood to be in $\{1, \ldots, n\}$. Fix $l \in\{1, \ldots, n\}$.

From condition (i) of the proposition it follows that $u_{m, 1}^{(\mu)} \sim \hat{u}_{m}$ on $\Gamma_{\mu}$. Thus the functions $f_{m} u_{m, 1}^{(\mu)}, m \in \mathrm{St}^{-}(\mu, l)$, together with the functions $f_{m} u_{m}^{(\mu+1)}, m \notin \mathrm{St}^{-}(\mu, l)$, form a fundamental system of solutions according 
to Proposition 9. Hence, there exist 1-periodic analytic functions $p_{m}^{(\mu)}, p_{m}^{(\mu+1)}$, $1 \leq m \leq n$, such that

$$
\begin{aligned}
u_{l}^{(\mu+1)}-u_{l, 1}^{(\mu)}=\sum_{m \in \mathrm{St}^{-}(\mu, l)} p_{m}^{(\mu)} f_{m l} u_{m, 1}^{(\mu)} & +\sum_{m \in \mathrm{St}^{+}(\mu, l)} p_{m}^{(\mu+1)} f_{m l} u_{m}^{(\mu+1)} \\
& +\sum_{m \notin \operatorname{St}(\mu, l)} p_{m}^{(\mu+1)} f_{m l} u_{m}^{(\mu+1)}
\end{aligned}
$$

We have $u_{l}^{(\mu+1)}-u_{l, 1}^{(\mu)}=u_{l}^{(\mu+1)}-u_{l}^{(\mu)}+u_{l}^{(\mu)}-u_{l, 1}^{(\mu)} \in(\mathcal{A} \leq-k)^{n}\left(H_{\mu} \cap H_{\mu+1}\right)$, and thus (by Lemma 7) we may conclude that each term in the sums on the right-hand side of (5.6) belongs to this set. Next we apply Lemma 8 to these terms, and find:

- If $m \in \mathrm{St}^{-}(\mu, l)$ (resp. $m \in \mathrm{St}^{+}(\mu, l)$ ): Then $f_{m l}$ is of level $k<1$, and it is an element of $\mathcal{A}^{\leq-k}\left(H_{\mu} \cap H_{\mu+1}\right)$. So there exist complex constants $c_{m}^{(\mu)}$ (resp. $\left.d_{m}^{(\mu+1)}\right)$ such that $\left(p_{m}^{(\mu)}(s)-c_{m}^{(\mu)}\right) f_{m l}(s)$ (resp. $\left.\left(p_{m}^{(\mu+1)}(s)-d_{m}^{(\mu+1)}\right) f_{m l}(s)\right)$ belong to $\mathcal{A}^{\leq-1}(H)$, where $H$ is the upper or lower half plane containing $H_{\mu} \cap H_{\mu+1}$.

- If $m \notin \operatorname{St}(\mu, l)$ : Then $\kappa_{m l}<k$ or $\kappa_{m l}=k$ with $f_{m l} \notin \mathcal{A}^{\leq-k}\left(H_{\mu} \cap H_{\mu+1}\right)$ or $\kappa_{m l}>k$ with in all cases $p_{m}^{(\mu+1)} f_{m l} \in \mathcal{A}^{\leq-k}\left(H_{\mu} \cap H_{\mu+1}\right)$. In the last case Lemma 8 implies $p_{m}^{(\mu+1)} f_{m l} \in \mathcal{A}^{\leq-k^{\prime}}\left(H_{\mu} \cap H_{\mu+1}\right)$. This also follows in the first two cases from Lemma 8-2 with $c=0$.

So we have

$$
u_{l}^{(\mu+1)}-u_{l, 1}^{(\mu)}=\sum_{m \in \mathrm{St}^{-}(\mu, l)} c_{m}^{(\mu)} f_{m l} u_{m, 1}^{(\mu)}+\sum_{m \in \mathrm{St}^{+}(\mu, l)} d_{m}^{(\mu+1)} f_{m l} u_{m}^{(\mu+1)}+\psi_{l, 1}^{(\mu)},
$$

with $\psi_{l, 1}^{(\mu)}$ a function in $\left(\mathcal{A}^{\leq-k^{\prime}}\right)^{n}\left(H_{\mu} \cap H_{\mu+1}\right)$. Obviously, if we define

$$
u_{l, 1}^{(\mu+1)}:=u_{l}^{(\mu+1)}-\sum_{m \in \mathrm{St}^{+}(\mu, l)} d_{m}^{(\mu+1)} f_{m l} u_{m}^{(\mu+1)},
$$

then $u_{l, 1}^{(\mu+1)}$ satisfies the requirements.

So, if we have constructed $u_{m, 1}^{(\lambda)}$ for $m \in\{1, \ldots, n\}$ and $\lambda \in\{M, M+$ $1, \ldots, \mu\}(\mu \leq N-1)$, then we can construct $u_{l, 1}^{(\mu+1)}$ for each $l \in\{1, \ldots, n\}$ and the lemma follows by induction on $\mu$.

We next refine the solutions of the previous lemma to solutions which satisfy (5.1) and (5.2) in Proposition 10.

Lemma 12. Let $k<1$. Suppose the assumptions of Proposition 10 hold, and furthermore, assume that $u_{l}^{(\mu+1)}-u_{l}^{(\mu)}=\sum_{m \in S t^{-}(\mu, l)} c_{l m}^{(\mu)} f_{m l} u_{m}^{(\mu)}+\psi_{l}^{(\mu)}$, for some constants $c_{l m}^{(\mu)}$ and a function $\psi_{l}^{(\mu)} \in\left(\mathcal{A}^{\leq-k^{\prime}}\right)^{n}\left(H_{\mu} \cap H_{\mu+1}\right)$. 
Then there exist fundamental systems $\left\{f_{l} \tilde{u}_{l}^{(\mu)}\right\}_{l=1}^{n}, \mu=M, \ldots, N$, such that

(i) $\tilde{u}_{l}^{(\mu)}-u_{l}^{(\mu)}=\sum_{m \in S t^{-}(\mu, l)} \tilde{c}_{l m}^{(\mu)} f_{m l} u_{m}^{(\mu)}$ for some constants $\tilde{c}_{l m}^{(\mu)}$;

(ii) $\tilde{u}_{l}^{(\mu+1)}-\tilde{u}_{l}^{(\mu)} \in\left(\mathcal{A} \leq-k^{\prime}\right)^{n}\left(H_{\mu} \cap H_{\mu+1}\right)$.

Proof. This lemma can also be proven by induction, but this time we start from the other end of the covering $\left\{\Gamma_{\mu}\right\}_{\mu=M}^{N}$ of $S(\alpha, \beta)$ : define $\tilde{u}_{m}^{(N)}:=u_{m}^{(N)}$ for all $m \in\{1, \ldots, n\}$. Next suppose $\tilde{u}_{m}^{(N)}, \ldots, \tilde{u}_{m}^{(\mu+1)}$ have been defined and possess the properties of the lemma for all $m \in\{1, \ldots, n\}$ and some $\mu \in\{M, \ldots, N-1\}$. Let $l \in\{1, \ldots, n\}$. We have

$$
\begin{aligned}
& \tilde{u}_{l}^{(\mu+1)}-u_{l}^{(\mu)} \\
& =\tilde{u}_{l}^{(\mu+1)}-u_{l}^{(\mu+1)}+u_{l}^{(\mu+1)}-u_{l}^{(\mu)} \\
& =\sum_{m \in \mathrm{St}^{-}(\mu+1, l)} \tilde{c}_{l m}^{(\mu+1)} f_{m l} u_{m}^{(\mu+1)}+\sum_{m \in \mathrm{St}^{-}(\mu, l)} c_{l m}^{(\mu)} f_{m l} u_{m}^{(\mu)}+\psi_{l}^{(\mu)} .
\end{aligned}
$$

Furthermore,

$$
u_{m}^{(\mu+1)}-u_{m}^{(\mu)}=\sum_{j \in \mathrm{St}^{-}(\mu, m)} c_{m j}^{(\mu)} f_{j m} u_{j}^{(\mu)}+\psi_{m}^{(\mu)} .
$$

From these two relations and properties (5.3) and (5.4) we obtain

$$
\tilde{u}_{l}^{(\mu+1)}-u_{l}^{(\mu)}=\sum_{m \in \mathrm{St}^{-}(\mu, l)} \tilde{c}_{l m}^{(\mu)} f_{m l} u_{m}^{(\mu)}+\tilde{\psi}_{l}^{(\mu)},
$$

where the $\tilde{c}_{l m}^{(\mu)}$ are constants in $\mathbf{C}$ and $\tilde{\psi}_{l}^{(\mu)} \in\left(\mathcal{A}^{\leq-k^{\prime}}\right)^{n}\left(H_{\mu} \cap H_{\mu+1}\right)$.

If we define

$$
\tilde{u}_{l}^{(\mu)}:=u_{l}^{(\mu)}+\sum_{m \in \mathrm{St}^{-}(\mu, l)} \tilde{c}_{l m}^{(\mu)} f_{m l} u_{m}^{(\mu)},
$$

then $\tilde{u}_{l}^{(\mu)}$ satisfies the requirements of the lemma. Again the lemma follows by induction on $\mu$.

The first statement of Proposition 10 follows from the previous lemmas and the last statement is a direct consequence of the relative Watson lemma referred to after Definition 2. We could also prove the uniqueness directly, without reference to this lemma, along the same lines as in [BIS].

Proof for $\mathbf{k}=1$.

Recall that the Stokes intervals of level 1 of Equation (1.1) with formal solution (2.3) are, by definition, the Stokes intervals of the functions $e^{2 \pi i s j} f_{m l}(s), j \in \mathbf{Z}, l, m \in\{1, \ldots, n\}$, that are of level 1 . Taking $l=m$ and 
$j \neq 0$, we find that $[(h-1) \pi, h \pi]$ is a Stokes interval of level 1 of the equation for any $h \in \mathbf{Z}$. Hence, due to the assumption that $(\alpha, \beta)$ does not contain a Stokes interval of level 1 , the sector $S(\alpha, \beta)$ does not contain a lift of both the positive and the negative real axis. From this and $\alpha<M \pi / 2<N \pi / 2<\beta$ it follows that $N-M \leq 2$ and at least one of the integers $M$ and $N$ has to be odd.

We will prove the proposition for the cases $M=-1$ and $N=0$ or $N=1$, that is $\alpha \in[-\pi,-\pi / 2), \beta \in(0, \pi]$. The other cases can be proven similarly.

Let $l \in\{1, \ldots, n\}$. There exist 1-periodic analytic functions $p_{m}^{(-1)}(s)$, $m=1, \ldots, n$, such that

$$
u_{l}^{(0)}-u_{l}^{(-1)}=\sum_{m=1}^{n} p_{m}^{(-1)} f_{m l} u_{m}^{(-1)} .
$$

By assumption (ii) of Proposition 10, we have $u_{l}^{(0)}-u_{l}^{(-1)} \in\left(\mathcal{A}^{\leq-1}\right)^{n}(-\pi / 2,0)$. So, by Lemma $7, p_{m}^{(-1)} f_{m l} \in \mathcal{A}^{\leq-1}(-\pi / 2,0), m \in\{1, \ldots, n\}$. From Lemma 8 we conclude that

$$
p_{m}^{(-1)} f_{m l} \in \mathcal{A}^{\leq-1}(-\pi, 0) \text { if } \kappa_{m l}<1,
$$

and

$$
p_{m}^{(-1)} f_{m l} \in \mathcal{A}^{\leq-1^{+}}(-\pi / 2,0) \text { if } \kappa_{m l}=1^{+} .
$$

If $\kappa_{m l}=1$ and $p_{m}^{(-1)} \neq 0$ then according to Lemma $8-3$ we have $\Im b_{m l} \geq 0$. Moreover, this lemma tells us that

$$
p_{m}^{(-1)} f_{m l} \in \mathcal{A}^{\leq-1}(-\pi, 0) \text { if } \kappa_{m l}=1, \Im b_{m l}=0 .
$$

Let $t_{1}, \ldots, t_{\nu}$ denote the numbers $\Im b_{h}, h \in\{1, \ldots, n\}$ in decreasing order of magnitude. We will use induction on $\tau \in\{1, \ldots, \nu\}$.

If $\Im b_{l}=t_{1}$, then $\Im b_{m l} \leq 0$ for all $m \in\{1, \ldots, n\}$. So if $\kappa_{m l}=1$ and $p_{m}^{(-1)} \neq 0$ then we know already that $\Im b_{m l} \geq 0$ and so $\Im b_{m l}=0$ and (5.10) applies. Therefore if $\Im b_{l}=t_{1}$ we define

$$
\tilde{u}_{l}^{(-1)}=u_{l}^{(-1)}+\sum_{\kappa_{m l} \leq 1} p_{m}^{(-1)} f_{m l} u_{m}^{(-1)}, \tilde{u}_{l}^{(0)}=u_{l}^{(0)} .
$$

Then from (5.7), (5.8), (5.9) and (5.10) it follows that $f_{l} \tilde{u}_{l}^{(\mu)}$ are solutions of (1.1) with

$$
\begin{aligned}
& \tilde{u}_{l}^{(\mu)}-u_{l}^{(\mu)} \in\left(\mathcal{A}^{\leq-1}\right)^{n}\left(\Gamma_{\mu}\right) \text { if } \mu \in\{-1,0\} ; \\
& \tilde{u}_{l}^{(0)}-\tilde{u}_{l}^{(-1)} \in\left(\mathcal{A}^{\leq-1^{+}}\right)^{n}(-\pi / 2,0) .
\end{aligned}
$$

Next let $\tau \in\{2, \ldots, \nu\}, \Im b_{l}=t_{\tau}$ and suppose that for all $m \in I(l):=$ $\left\{m \in\{1, \ldots, n\} \mid \kappa_{m l}=1, \Im b_{m l}>0\right\}$ the functions $\tilde{u}_{m}^{(-1)}$ and $\tilde{u}_{m}^{(0)}$ have 
already been defined such that (5.11) holds with $l$ replaced by $m$. We have

$$
u_{l}^{(0)}-u_{l}^{(-1)}=\sum_{m \notin I(l)} \tilde{p}_{m}^{(-1)} f_{m l} \tilde{u}_{m}^{(-1)}+\sum_{m \in I(l)} \tilde{p}_{m}^{(-1)} f_{m l} \tilde{u}_{m}^{(-1)},
$$

for some 1-periodic functions $\tilde{p}_{m}^{(-1)}(s)$ analytic on a neighbourhood of $\infty$ in $H_{-1}$. As before we have

$$
\tilde{p}_{m}^{(-1)} f_{m l} \in \mathcal{A}^{\leq-1}(-\pi / 2,0) .
$$

Now we have analogues of (5.8), (5.9) and (5.10), and so the first sum can be written as $\varphi_{l}^{(-1)}+\psi_{l}^{(-1)}$ such that $\varphi_{l}^{(-1)} \in\left(\mathcal{A}^{\leq-1}\right)^{n}\left(H_{-1}\right)$ and $\psi_{l}^{(-1)} \in$ $\left(\mathcal{A}^{\leq-1^{+}}\right)^{n}(-\pi / 2,0)$ and $f_{l} \varphi_{l}^{(-1)}$ and $f_{l} \psi_{l}^{(-1)}$ are solutions of (1.1).

Next consider the case that $m \in I(l)$ and $\tilde{p}_{m}^{(-1)} \neq 0$. Then according to Lemma 8-3 there exist analytic 1-periodic functions $p_{m}^{ \pm}$such that $\tilde{p}_{m}^{(-1)}=$ $p_{m}^{-}+p_{m}^{+}$and $p_{m}^{-} f_{m l} \in \mathcal{A}^{\leq-1}(\alpha, 0)$ and $p_{m}^{+} f_{m l} \in \mathcal{A}^{\leq-1}(-\pi / 2, \beta)$. Now define

$$
\begin{gathered}
\tilde{u}_{l}^{(-1)}:=u_{l}^{(-1)}+\varphi_{l}^{(-1)}+\sum_{m \in I(l)} p_{m}^{-} f_{m l} \tilde{u}_{m}^{(-1)}, \\
\tilde{u}_{l}^{(0)}:=u_{l}^{(0)}-\sum_{m \in I(l)} p_{m}^{+} f_{m l} \tilde{u}_{m}^{(0)} .
\end{gathered}
$$

Then $\tilde{u}_{l}^{(0)}-\tilde{u}_{l}^{(-1)}=\psi_{l}^{(-1)}+\sum_{m \in I(l)} p_{m}^{+} f_{m l}\left(\tilde{u}_{m}^{(-1)}-\tilde{u}_{m}^{(0)}\right) \in\left(\mathcal{A}^{\leq-1^{+}}\right)^{n}(-\pi / 2,0)$ and it follows that the functions $\tilde{u}_{l}^{(\mu)}$ satisfy (5.11). By induction (5.11) follows for all $l$. So in case $N=0$ the proposition has been proved.

Next suppose that $N=1$, so $\beta>\pi / 2$. If $p_{m}^{(1)}(s), m=1, \ldots, n$, are the 1-periodic functions analytic on a neighbourhood of $\infty$ in the upper half plane such that

$$
u_{l}^{(1)}-\tilde{u}_{l}^{(0)}=\sum_{m=1}^{n} p_{m}^{(1)} f_{m l} u_{m}^{(1)},
$$

then $p_{m}^{(1)} f_{m l} \in \mathcal{A}^{\leq-1}(0, \pi / 2)$. If $\kappa_{m l}<1$ then as before $p_{m}^{(1)} f_{m l} \in \mathcal{A}^{\leq-1}\left(H_{1}\right)$. Next suppose $\kappa_{m l}=1$ and $p_{m}^{(1)} \neq 0$. Then by Lemma 8-3 there exists an integer $N$ such that $p_{m}^{(1)}(s)=p_{N} e^{2 \pi i s N}(1+o(1))$ as $\Im s \rightarrow \infty$ with $p_{N} \neq 0$ and therefore $e^{2 \pi i s N} f_{m l}(s) \in \mathcal{A}^{\leq-1}(0, \pi / 2)$. So there exists a Stokes interval $\left[\sigma_{m}-\pi, \sigma_{m}\right]$ of $e^{2 \pi i s N} f_{m l}$ which contains $(0, \pi / 2)$. Now $\sigma_{m}-\pi \geq-\pi / 2>\alpha$ and therefore $\sigma_{m}>\beta$. Hence $p_{m}^{(1)} f_{m l} \in \mathcal{A}^{\leq-1}(0, \beta)$. Moreover, if $\kappa_{m l}=1^{+}$, then $p_{m}^{(1)} f_{m l} \in \mathcal{A}^{\leq-1^{+}}(0, \pi / 2)$ according to Lemma 8. Thus, if we define $\tilde{u}_{l}^{(1)}:=u_{l}^{(1)}-\sum_{\kappa_{m l} \leq 1} p_{m}^{(1)} f_{m l} u_{m}^{(1)}$, then

$$
\tilde{u}_{l}^{(1)}-u_{l}^{(1)} \in\left(\mathcal{A}^{\leq-1}\right)^{n}(0, \beta) ; \tilde{u}_{l}^{(1)}-\tilde{u}_{l}^{(0)} \in\left(\mathcal{A}^{\leq-1^{+}}\right)^{n}(0, \pi / 2) .
$$


The fundamental systems $\left\{f_{l} \tilde{u}_{l}^{(\mu)}\right\}_{l=1}^{n}, \mu=-1,0,1$, thus obtained satisfy (5.1) and (5.2).

The uniqueness property of Proposition 10 is an immediate consequence of a more general form of the relative Watson lemma by Malgrange and Ramis, that can be found in [BIS].

\section{Equations without level $1^{+}$.}

Theorem 13 has already been stated and proven in [BF96], but here we present a new proof.

Theorem 13. Let $0<k_{1}<\cdots<k_{r}=1$ be the levels in $(0,1]$ of Equation (1.1), and suppose that this equation does not contain a level $1^{+}$(i.e., $d_{m l}=$ $0, \forall m, l \in\{1, \ldots, n\})$. Let $\hat{\mathrm{H}}(s) s^{\Lambda s} e^{\mathrm{G}(s)} s^{\mathrm{L}}$ be a formal fundamental matrix as in (1.2).

Let $S_{i}=S\left(\alpha_{i}, \beta_{i}\right), i=1, \ldots, r$, be a sequence of open sectors such that $S_{1} \supset \ldots \supset S_{r}, S_{1}$ has aperture less than $2 p \pi, S_{i}$ has aperture larger than $\pi / k_{i}$ and $\left(\alpha_{i}, \beta_{i}\right)$ does not contain a Stokes interval of level $k_{i}, i=1, \ldots, r$.

Then $\hat{\mathrm{H}}$ is $\left(k_{1}, \ldots, k_{r}\right)$-summable on $\left(S_{1}, \ldots, S_{r}\right)$ with sum $\mathrm{H}_{r}$ such that $\mathrm{H}_{r}(s) s^{\Lambda s} e^{\mathrm{G}(s)} s^{\mathrm{L}}$ is an analytic fundamental matrix of (1.1).

Proof. Define $M_{j}, N_{j}, j=1, \ldots, r$, to be the integers such that $\left(M_{j}-\right.$ 1) $\pi / 2 \leq \alpha_{j}<M_{j} \pi / 2<N_{j} \pi / 2<\beta_{j} \leq\left(N_{j}+1\right) \pi / 2$ and let $\Gamma_{j, \mu}:=S_{j} \cap H_{\mu}$, $\mu=M_{j}, \ldots, N_{j}, j=1, \ldots, r$. Also, let $S_{0}:=\mathbf{C}_{p}$ be the Riemann surface of $s^{1 / p}, \Gamma_{0, \mu}:=H_{\mu}, \mu=M_{0}, \ldots, N_{0}$ where $M_{0}:=M_{1}, N_{0}:=M_{0}+4 p-1$.

By Proposition 9 we have a representative $\left\{\mathrm{H}^{(\mu)}(s)\right\}_{\mu=M_{0}}^{N_{0}}$ of $T^{-1} \hat{\mathrm{H}}(s)$ on the covering $\left\{H_{\mu}\right\}_{\mu=M_{0}}^{N_{0}}$ of $\mathbf{C}_{p}$ such that $\mathrm{H}^{(\mu)}(s) s^{\Lambda s} e^{\mathrm{G}(s)} s^{\mathrm{L}}$ is an analytic fundamental matrix of (1.1). To show that the columns $\hat{h}_{l}$ of $\hat{\mathrm{H}}$ are multisummable we have to construct $h_{l, j} \in\left(\mathcal{A}_{\left(1 / k_{1}\right)} / \mathcal{A}^{\leq-k_{j+1}}\right)^{n}\left(S_{j}\right), j=0, \ldots, r$ such that $\left.h_{l, j}\right|_{S_{j+1}} \equiv h_{l, j+1} \bmod \mathcal{A}^{\leq-k_{j+1}}, j=0, \ldots, r$ if $k_{r+1}=\infty$.

Let $\mathrm{U}_{0}^{(\mu)}(s):=\mathrm{H}^{(\mu)}(s) s^{\mathrm{N}}$ so that $\mathrm{U}_{0}^{(\mu)}(s) \mathrm{F}(s)$ is a fundamental matrix of (1.1) (cf. $(2.2))$ and let $\tilde{u}_{l, 0}^{(\mu)}$ denote the $l$ th column of $\mathrm{U}_{0}^{(\mu)}$. The construction mentioned above is equivalent to the construction of functions $\left\{\tilde{u}_{l, j}^{(\mu)}\right\}_{\mu=M_{j}}^{N_{j}}$ for $j=1, \ldots, r$ and $l=1, \ldots, n$ such that:

(1) $\left\{f_{l} \tilde{u}_{l, j}^{(\mu)}\right\}_{l=1}^{n}$ is a fundamental system of Equation (1.1),

(2) $\left\{\tilde{u}_{l, j}^{(\mu)}\right\}_{\mu=M_{j}}^{N_{j}}$ represents a $k_{j+1}$-precise quasi-function $\tilde{u}_{l, j} \in\left(\mathcal{A}_{\left(1 / k_{1}\right)} / \mathcal{A}^{\leq-k_{j+1}}\right)^{n}\left(S_{j}\right)[\log s], j=1, \ldots, r$,

(3) $\left.\tilde{u}_{l, j-1}\right|_{S_{j}} \equiv \tilde{u}_{l, j} \bmod \left(\mathcal{A} \leq-k_{j}\right)^{n}, j=1, \ldots, r$.

Suppose we have constructed $\tilde{u}_{l, i}$ for $i=0, \ldots, j-1$, for some $j \in$ $\{1, \ldots, r\}$. Then we can apply Proposition 10 with $\alpha=\alpha_{j}, \beta=\beta_{j}$, and 
with $u_{l}^{(\mu)}=\left.\tilde{u}_{l, j-1}^{(\mu)}\right|_{\Gamma_{j, \mu}}, \mu=M_{j}, \ldots, N_{j}$. Defining $\tilde{u}_{l, j}^{(\mu)}:=\tilde{u}_{l}^{(\mu)}, l=1, \ldots, n$, $\mu=M_{j}, \ldots, N_{j}$ we see that properties (1), (2) and (3) are satisfied for $i=j$ as well. So they are satisfied for all $j \in\{1, \ldots, r\}$.

We have $\tilde{u}_{l, r}^{(\mu+1)}-\tilde{u}_{l, r}^{(\mu)} \in\left(\mathcal{A}^{\leq-1^{+}}\right)^{n}\left(\Gamma_{r, \mu} \cap \Gamma_{r, \mu+1}\right)$. We also have $\tilde{u}_{l, r}^{(\mu+1)}-$ $\tilde{u}_{l, r}^{(\mu)}=\sum_{m=1}^{n} p_{m} f_{m l} \tilde{u}_{m, r}^{(\mu)}$ for some 1-periodic analytic functions $p_{m}$. Lemma 7 now tells us that each $p_{m} f_{m l} \in \mathcal{A}^{\leq-1^{+}}\left(\Gamma_{\mu, r} \cap \Gamma_{\mu+1, r}\right)$, and then it follows from Lemma 8 and the fact that the equation has no level $1^{+}$, that $p_{m}=0$, for all $m$. Hence, the functions $\tilde{u}_{l, r}^{(\mu)}, \mu=M_{r}, \ldots, N_{r}$, are the restrictions of an analytic function $\tilde{u}_{l, r} \in\left(\mathcal{A}_{\left(1 / k_{1}\right)}\right)^{n}\left(S_{r}\right)[\log s]$.

\section{Equations with level $1^{+}$.}

In this section we will consider Equation (1.1) under the assumption that there does exist a pair $(m, l)$ such that $d_{m} \neq d_{l}$ in the notation of $(2.3)$; that is, the equation possesses the level $1^{+}$. We will show in this section that we can still assign a uniquely characterizable fundamental system $Y(s)$ with asymptotic expansion $\hat{Y}(s)$ in appropriate regions of the Riemann surface of the logarithm, provided $\Re b_{l} \neq \Re b_{m}$ if $b_{l} \neq b_{m}$ (cf. notation in (2.3)).

Before we state the main result of this paper (Theorem 18), we need to define the Stokes numbers of level $1^{+}$of the equation. The Stokes number of a function $f$ of level $1^{+}$of the form

$$
f(s)=\exp (d s \log s+2 \pi i b s+q(s)+\gamma \log s), \text { with } d \neq 0,
$$

occurring in formal solutions of equations possessing a level $1^{+}$, is associated with curves that separate regions of growth from regions of decay of $f$. We have

$\Re\{d s \log s+2 \pi i b s\}=d \Re s \log |s|-(d \arg s+2 \pi \Re b) \Im s-2 \pi \Im b \Re s$,

and therefore the main contribution to $|f(s)|$ comes from $\exp [\Re\{s(d \log s+$ $2 \pi i \Re b)\}]$. Let $h$ be an even integer if $d<0$ and an odd integer if $d>0$. Then $f \in \mathcal{A}^{\leq-1^{+}}\left(H_{2 h}\right)$. Moreover, $f$ behaves as an exponential function of order 1 in vertical strips, $f$ becomes exponentially large on any open sector containing $\bar{H}_{2 h}$ and, if $S_{+}(h):=S\left(\left(h-\frac{1}{2}\right) \pi,\left(h+\frac{1}{2}\right) \pi\right]$ and $S_{-}(h):=$ $S\left[\left(h-\frac{1}{2}\right) \pi,\left(h+\frac{1}{2}\right) \pi\right)$ then it is easily verified that

$$
f \in \mathcal{A}^{\leq-1}\left(S_{ \pm}\right) \text {iff } \pm(h+2 \Re b / d)<-1 / 2,
$$

where the upper (lower) signs belong together.

Let $\left\{f_{j} u_{j}\right\}_{j=1}^{n}$ be a fundamental system of (1.1) such that $u_{j} \sim \hat{u}_{j}$ as $s \rightarrow \infty$ on $S_{ \pm}(h)$. Assume $d_{m l}=d_{m}-d_{l}<0$ and $h$ is even. Then there exists $N_{ \pm} \in \mathbf{Z}$ such that $\pm\left\{h+2\left(\Re b_{m l}+N_{ \pm}\right) / d_{m l}\right\}<-1 / 2$. Then $e^{2 \pi i s N_{ \pm}} f_{m l} \in$ $\mathcal{A}^{\leq-1}\left(S_{ \pm}\right)$and therefore the solutions $f_{l} u_{l}$ and $f_{l} u_{l}+e^{2 \pi i s N_{ \pm}} f_{m} u_{m}$ have the same asymptotic behaviour on $S_{ \pm}(h)$. So in this case it is not possible to 
characterize fundamental systems $\mathrm{Y}(s)$ by their asymptotic behaviour $\hat{\mathrm{Y}}(s)$ on $S_{ \pm}(h)$.

In order for a fundamental system to be in some way uniquely determined by its asymptotic expansion in a sector of $\mathbf{C}_{\infty}$, which contains an open right or left half plane, this sector therefore should contain the closure of this half plane. However, such fundamental systems do not exist in general (see [vdPS97, Chapter 11]).

Hence we have to characterize fundamental systems by their asymptotic behaviour in a more complicated type of region. This region should contain a neighbourhood of $\infty$ in some half plane $H_{2 h}$ but not a neighborhood of $\infty$ in $\bar{H}_{2 h}$. In the case $d=(-1)^{h+1}$ a suitable region is given by

Definition 14. For $\theta \in \mathbf{R}, h \in \mathbf{Z}$, we define

$$
\begin{aligned}
D(h ; \theta):=\left\{s \in \mathbf{C}_{\infty} \mid(h-1) \pi<\arg s<(h+1) \pi ;\right. \\
\left.(-1)^{h} \Re\{s(\log s+i \theta)\}>1\right\} .
\end{aligned}
$$

If $h \in \mathbf{Z}$ and $\theta_{1}, \theta_{2} \in \mathbf{R}$ then $D\left(h ; \theta_{1}, \theta_{2}\right):=D\left(h ; \theta_{1}\right) \cup D\left(h ; \theta_{2}\right)$.

We denote the boundary of $D(h ; \theta)$ by $C(h ; \theta)$. We have $D(h ; \theta) \subset$ $\bigcup_{j=-1}^{1} H_{2 h+j}$ and $s e^{i h \pi} \in D(h ; \theta) \Leftrightarrow s \in D(0 ; \theta+h \pi)$. Similarly with $D$ replaced by $C$. Details on $C(0 ; \theta)$ can be found in [Imm84] and [Imm91]. We have $\Re s=O(\Im s / \log |s|)$ as $s \rightarrow \infty$ on $C(h ; \theta)$. This implies that $\arg s \rightarrow \pm \pi / 2 \bmod 2 \pi$ as $\Im s \rightarrow \pm \infty, s \in C(h ; \theta)$.

If $h \in \mathbf{Z}$ and $\theta \in \mathbf{R}$, then the following properties are easily established.

- Let $\tilde{\theta}<\theta$. As $(-1)^{h} \Re\{s(\log s+i \tilde{\theta})\}=(-1)^{h} \Re\{s(\log s+i \theta)\}-$ $(-1)^{h}(\tilde{\theta}-\theta) \Im s$, it follows that:

i) $D(h ; \tilde{\theta}) \cap H_{2 h-1} \subset D(h ; \theta) \cap H_{2 h-1}$, and that

ii) $D(h ; \tilde{\theta}) \cap H_{2 h+1} \supset D(h ; \theta) \cap H_{2 h+1}$.

One could say that the regions $D(h ; \theta)$ 'rotate' (modulo some deformation) clockwise with increasing $\theta$.

- $D(h ; \theta) \cap H_{2 h}$ is a neighbourhood of $\infty$ in $H_{2 h}$. However, since for any $s_{0} \in \bar{H}_{2 h}, s_{0}+\bar{H}_{2 h} \not \subset D(h ; \theta)$, the intersection $D(h ; \theta) \cap \bar{H}_{2 h}$ is not a neighbourhood of $\infty$ in $\bar{H}_{2 h}$.

Let $D:=D\left(h ; \theta_{1}, \theta_{2}\right)$, for some $h \in \mathbf{Z}$ and $\theta_{1}<\theta_{2}$. A set $U \subset D$ is called a neighbourhood of $\infty$ in $D$, if, for any $\theta \in\left(\theta_{1}, \theta_{2}\right)$, there exists $s_{0} \in D$ such that $s_{0}+D(h ; \theta) \subset U$.

Suppose $g$ is an analytic function on a neighbourhood $U$ of $\infty$ in $D$, and suppose there exist a $k>0$ and a series $\hat{g}(s)=\sum_{n \geq 0} g_{n} s^{-n / p}$ such that for any $\theta \in\left(\theta_{1}, \theta_{2}\right)$ we have

$$
\left|g(s)-\sum_{n=0}^{N-1} g_{n} s^{-n / p}\right| \leq K A^{N} \Gamma\left(\frac{N}{p k}\right)|s|^{-N / p},
$$




$$
\forall s \in D(h ; \theta) \cap U, \forall N \in \mathbf{N},
$$

for some positive constants $K$ and $A$, which only depend on $\theta$. Then we call $g$ a $k$-Gevrey function on $D$ (with respect to $\frac{1}{p} \mathbf{N}_{0}$ ), and the set of these functions is denoted by $\mathcal{A}_{(1 / k)}(D)$.

Concerning 1-Gevrey functions on $D$ we have the following theorem by Immink (cf. [Imm96]):

Theorem 15. Suppose $\theta_{1}, \theta_{2} \in \mathbf{R}, \theta_{1}<\theta_{2}$, and $h \in \mathbf{Z}$. Let $D:=$ $D\left(h ; \theta_{1}, \theta_{2}\right)$.

If $g \in \mathcal{A}_{(1)}(D)$, then $g$ is uniquely determined by its asymptotic expansion $\hat{g}$.

In [Imm96] it is shown that $g$ is already uniquely determined by its asymptotic expansion if (7.2) with $k=1$ holds on $D(h ; \theta)$ for one $\theta \in\left(\theta_{1}, \theta_{2}\right)$.

Let $f$ be a function of level $1^{+}$given by (7.1) and $\theta_{1}<2 \pi \Re b / d<\theta_{2}$. Let $h \in \mathbf{Z}$ be odd if $d>0$ and even if $d<0$. Then there exist positive constants $K$ and $c$ such that $|f(s)| \leq K e^{-c|s|}$ for $s \in D\left(h ; \theta_{1}\right) \cap D\left(h ; \theta_{2}\right)$ and $|s|$ sufficiently large. From this it follows that (7.2) holds on this set with $g:=f, g_{n}=0$, and $k=1$. This is not true if $\frac{2 \pi \Re b}{d} \notin\left[\theta_{1}, \theta_{2}\right]$. Therefore we introduce the following definition:

Definition 16. Let $f(s)$ be the function of level $1^{+}$given by (7.1). Then we call $\frac{2 \pi \Re b}{d}$ its Stokes number. The Stokes numbers of level $1^{+}$of the equation (1.1) are the Stokes numbers of the functions $e^{2 \pi i j s} f_{m l}(s)$, $l, m \in\{1, \ldots, n\}, j \in \mathbf{Z}$, that are of level $1^{+}$. That is, they are given by the expression

$$
\frac{2 \pi}{d_{m l}}\left(\Re b_{m l}+j\right), j, l, m \text { as above, } d_{m l} \neq 0 .
$$

Suppose $\theta_{1}<\theta_{2}$ and $\alpha<\beta$. Let $D:=D\left(h ; \theta_{1}, \theta_{2}\right)$ and $S:=S(\alpha, \beta)$. Assume $D \cap S \neq \emptyset$. We define a neighbourhood of $\infty$ in $D \cap S$ to be an open set $U$ in $D \cap S$, such that for any $\theta, \gamma, \delta$ satisfying $\theta_{1}<\theta<\theta_{2}$ and $\alpha<\gamma<\delta<\beta$, there exists $s_{0} \in D \cap S$ such that $s_{0}+(D(h ; \theta) \cap S(\gamma, \delta)) \subset$ $U$. We write $\mathcal{A}^{\leq-1}(D \cap S)$ for the set of functions that are analytic on a neighbourhood of $\infty$ in $D \cap S$, and exponentially small of order 1 , as $s \rightarrow \infty$, uniformly on $D(h ; \theta) \cap S(\gamma, \delta)$, for any $\theta, \gamma, \delta$ as above.

We extend Definition 2 of multisummability as follows:

Definition 17. Let $0<k_{1}<\cdots<k_{r-1}<k_{r}=1$, and define $k_{r+1}=1^{+}$. Let $S_{1} \supset \ldots \supset S_{r}$ be a nested sequence of sectors $S_{i}$ with aperture $>\pi / k_{i}$, $i=1, \ldots, r$, aperture of $S_{1}$ at most $2 p \pi$ and assume $S_{r} \supset S\left(\left(h-\frac{1}{2}\right) \pi-\right.$ $\left.\varepsilon,\left(h+\frac{1}{2}\right) \pi+\varepsilon\right)$, for some $h \in \mathbf{Z}$ and some $\varepsilon>0$. Finally, let $D:=D\left(h ; \theta_{1}, \theta_{2}\right)$ for some $\theta_{1}<\theta_{2}$.

A formal power series $\hat{f} \in \mathbf{C}\left[\left[s^{-1 / p}\right]\right]_{1 /\left(p k_{1}\right)}$ is called $\left(k_{1}, \ldots, k_{r}, 1^{+}\right)$summable on $\left(S_{1}, \ldots, S_{r}, D\right)$ with $\left(k_{1}, \ldots, k_{r}, 1^{+}\right)$-sum $f \in \mathcal{A}_{\left(1 / k_{1}\right)}(D)$, 
if there exist quasi-functions $f_{i} \in\left(\mathcal{A}_{\left(1 / k_{1}\right)} / \mathcal{A}^{\leq-k_{i+1}}\right)\left(S_{i}\right), i=1, \ldots, r$, satisfying:

$\left.f_{i}\right|_{S_{i+1}} \equiv f_{i+1} \bmod \mathcal{A}^{\leq-k_{i+1}}, i=0, \ldots, r-1$ with $f_{0}=T^{-1} \hat{f} ;$

$f_{r}$ has a representative $\left\{f_{r, \omega}\right\}_{\omega \in \Omega}$ with respect

to a covering $\left\{S_{r, \omega}\right\}_{\omega \in \Omega}$ of $S_{r}$ with open sectors $S_{r, \omega}$

such that $f_{r, \omega}-f \in \mathcal{A}^{\leq-1}\left(D \cap S_{r, \omega}\right), \forall \omega \in \Omega$.

Let $g$ be another function such that $f_{r, \omega}-g \in \mathcal{A}^{\leq-1}\left(D \cap S_{r, \omega}\right), \forall \omega \in \Omega$. Then $f-g \in \mathcal{A}^{\leq-1}\left(D \cap S_{r, \omega}\right), \forall \omega \in \Omega$, hence, $f-g \in \mathcal{A}^{\leq-1}\left(D \cap S_{r}\right)=$ $\mathcal{A}^{\leq-1}(D)$. Theorem 15 implies $f=g$ and it follows that $f$ is uniquely determined by $f_{r}$ and $D$. By the relative Watson lemma ([MR92, Prop. (2.1)]) $f_{i}$ is uniquely determined by $f_{i-1}$ and $S_{i}, i=r, \ldots, 1$. Hence the sum $f$ is uniquely determined by $\hat{f}$ and $\left(S_{1}, \ldots, S_{r}, D\right)$.

Similarly to Definition 2 we extend this definition to the case that $\hat{f}$ is an $n$-vector or $n \times n$-matrix with elements in $\mathbf{C}\left[\left[s^{-1 / p}\right]\right]_{1 /\left(p k_{1}\right)}$.

The main result of this paper is the following theorem.

Theorem 18. Let $k_{1}<\cdots<k_{r}=1$ be the sequence of positive levels $\leq 1$ of (1.1). Suppose that $1^{+}$is a level of (1.1) and that $\Re b_{l} \neq \Re b_{m}$ if $b_{l} \neq b_{m}$ where $b_{l}$ is defined below (2.3). Let $\hat{\mathrm{H}}(s) s^{\Lambda s} e^{\mathrm{G}(s)} s^{\mathrm{L}}$ be a formal fundamental matrix as in (1.2).

Let $S_{i}=S\left(\alpha_{i}, \beta_{i}\right), i=1, \ldots, r$, be a sequence of open sectors, such that $S_{1} \supset \ldots \supset S_{r}$ and $\beta_{1}-\alpha_{1} \leq 2 p \pi, \beta_{i}-\alpha_{i}>\pi / k_{i}$ and $\left(\alpha_{i}, \beta_{i}\right)$ does not contain a Stokes interval of level $k_{i}, i=1, \ldots, r$ of (1.1). Moreover, suppose that $(h-1) \pi<\alpha_{r}<\left(h-\frac{1}{2}\right) \pi$ and $\left(h+\frac{1}{2}\right) \pi<\beta_{r}<(h+1) \pi$, for some $h \in \mathbf{Z}$. Let $\tilde{\theta}, \theta \in \mathbf{R}, \tilde{\theta}<\theta$, such that $(\tilde{\theta}, \theta)$ does not contain a Stokes number of level $1^{+}$and define $D=D(h ; \tilde{\theta}, \theta)$.

Then $\hat{\mathrm{H}}$ is $\left(k_{1}, \ldots, k_{r}, 1^{+}\right)$-summable on $\left(S_{1}, \ldots, S_{r}, D\right)$ with $\left(k_{1}, \ldots\right.$, $\left.k_{r}, 1^{+}\right)$-sum $\mathrm{H}$ such that (1.3) defines an analytic fundamental matrix of $(1.1)$.

For the proof of this theorem we will use the following lemma which extends the results of Lemma 8-4.

Lemma 19. Let $h \in \mathbf{Z}$ and $Q_{ \pm}$be the quadrant $H_{2 h} \cap H_{2 h \pm 1}$. Here and in the following the upper signs belong together and so do the lower signs. Let $f$ be given by (7.1). Define $\theta_{j}:=\frac{2 \pi}{d}(\Re b+j), j \in \mathbf{Z}$, and $D:=D\left(h ; \theta_{N-1}, \theta_{N}\right)$, for some $N \in \mathbf{Z}$.

Suppose that $p(s) \not \equiv 0$ is a 1-periodic analytic function on a neighbourhood of $\infty$ in $H_{2 h \pm 1}$ such that $p(s) f(s) \in \mathcal{A}^{\leq-1^{+}}\left(Q_{ \pm}\right)$.

Then there exists a 1-periodic function $p_{-}(s)$, such that $p_{-}(s)$ is analytic on a neighbourhood of $\infty$ in $H_{2 h \pm 1}, p_{+}(s):=p(s)-p_{-}(s)$ is an entire 
function, and

$$
\begin{gathered}
p_{-}(s) f(s) \in \mathcal{A}^{\leq-1}\left(D \cap H_{2 h \pm 1}\right), \\
p_{+}(s) f(s) \in \mathcal{A}^{\leq-1}\left(D \cap H_{2 h \mp 1}\right) \cap \mathcal{A}^{\leq-1^{+}}\left(H_{2 h}\right) .
\end{gathered}
$$

Proof. We will give the details of the proof for the lower sign and $h$ is even. The other cases can be proven in a similar way.

Now $Q_{-}$is a fourth quadrant and $H:=H_{2 h-1}$ is a lower half plane. According to Lemma 8-4 we have $p(s) f(s) \in \mathcal{A}^{\leq-1^{+}}\left(\left(h-\frac{1}{2}\right) \pi, h \pi\right]$, and, since $p(s) \not \equiv 0$ also $f(s) \in \mathcal{A}^{\leq-1^{+}}\left(\left(h-\frac{1}{2}\right) \pi, h \pi\right)$. Therefore $d<0, f(s) \in$ $\mathcal{A}^{\leq-1^{+}}\left(\left(h-\frac{1}{2}\right) \pi,\left(h+\frac{1}{2}\right) \pi\right)$ and $\theta_{N}<\theta_{N-1}$.

We have an expansion for $p(s)$ as in (3.1) for $\Im s<-\rho$ for some $\rho>0$. Let $p_{-}(s):=\sum_{j \leq N-1} p_{j} e^{2 \pi i s j}$. Then $p_{-}(s)$ is analytic for $\Im s<-\rho$ and $p_{-}(s)=e^{2 \pi i s(N-1)} O(1), s \rightarrow \infty$ on $\bar{H}$. Moreover, $p_{+}(s)=\sum_{j \geq N} p_{j} e^{2 \pi i s j}$ is an entire function.

First consider $p_{-}(s) f(s)$. The properties of $p_{-}$imply

$$
p_{-}(s) f(s) \in \mathcal{A}^{\leq-1^{+}}\left(\left(h-\frac{1}{2}\right) \pi, h \pi\right] \subset \mathcal{A}^{\leq-1^{+}}\left(Q_{-}\right) .
$$

For $\varepsilon>0$, let $S_{\varepsilon}:=S\left(\left(h-\frac{1}{2}\right) \pi-\varepsilon,\left(h-\frac{1}{2}\right) \pi+\varepsilon\right)$. In order to prove that $p_{-}(s) f(s) \in \mathcal{A}^{\leq-1}(D \cap H)$ it is sufficient to show, that with any $\theta \in$ $\left(\theta_{N}, \theta_{N-1}\right)$ we can find positive constants $K, c$ and $\varepsilon$, such that

$$
\left|p_{-}(s) f(s)\right| \leq K e^{-c|s|}, s \in D(h ; \theta) \cap S_{\varepsilon}, \Im s<-\rho .
$$

As $p_{-}(s) f(s)=O(1) \exp (d s \log s+2 \pi i s(b+N-1)+o(s)), s \rightarrow \infty$ on $H$, it is sufficient to prove that

$$
\Re\{d s \log s+2 \pi i s(b+N-1)\} \leq-c|s|, \forall s \in D(h ; \theta) \cap S_{\varepsilon},
$$

for some $c>0$.

So let $\theta \in\left(\theta_{N}, \theta_{N-1}\right)$. On $D(h ; \theta)$ we have:

$$
\begin{aligned}
& \Re\{d s \log s+2 \pi i s(b+N-1)\} \\
& =d \Re\left\{s(\log s+i \theta)+i s\left(\frac{2 \pi}{d}(b+N-1)-\theta\right)\right\} \\
& <-d\left[\Im s\left(\theta_{N-1}-\theta\right)+\frac{2 \pi}{d} \Re s \Im b\right] \\
& =-d \sin (\arg s)\left(\theta_{N-1}-\theta\right)|s|\left(1+\frac{2 \pi \Im b}{d\left(\theta_{N-1}-\theta\right)} \cot (\arg s)\right) .
\end{aligned}
$$

We have $d \sin (\arg s)\left(\theta_{N-1}-\theta\right)>0$ on $H$. Furthermore, there exists an $\varepsilon>0$ such that $\left|\frac{4 \pi \Im b}{d\left(\theta_{N-1}-\theta\right)} \cot (\arg s)\right|<1, \forall s \in S_{\varepsilon}$. Thus (7.5) and (7.4) follow.

Next consider $p_{+}(s) f(s)$. As $p(s) f(s), p_{-}(s) f(s) \in \mathcal{A}^{\leq-1^{+}}\left(\left(h-\frac{1}{2}\right) \pi, h \pi\right]$, also $p_{+}(s) f(s)$ belongs to this set. Moreover, $f(s) \in \mathcal{A}^{\leq-1^{+}}\left(H_{2 h}\right)$ and $\left|p_{+}(s)\right| \leq K e^{-2 \pi N \Im s}, \Im s \geq-\rho$ for some $K>0$, and therefore $p_{+}(s) f(s) \in$ $\mathcal{A}^{\leq-1^{+}}\left(H_{2 h}\right)$. To prove the lemma it now suffices to prove that $p_{+} f \in$ 
$\mathcal{A}^{\leq-1}\left(D \cap H_{2 h+1}\right)$, hence that for any $\theta \in\left(\theta_{N}, \theta_{N-1}\right)$ we can find positive constants $K, c$ and $\varepsilon$, such that

$$
\left|p_{+}(s) f(s)\right| \leq K e^{-c|s|}, \forall s \in D(h ; \theta) \cap S\left(\left(h+\frac{1}{2}\right) \pi-\varepsilon,\left(h+\frac{1}{2}\right) \pi+\varepsilon\right) .
$$

A proof of this inequality runs along the same lines as that of (7.4).

The following proposition extends the results of Proposition 10.

Proposition 20. Let $k_{1}<\cdots<k_{r}$ be the positive levels $\leq 1$ of equation (1.1) and assume that (1.1) has a level $1^{+}$.

Let $M$ be odd. Assume $\alpha<M \pi / 2, \beta>(M+2) \pi / 2$ such that $I=$ $(\alpha, \beta)$ does not contain a Stokes interval of level 1 of (1.1). Define $\Gamma_{\mu}:=$ $H_{\mu} \cap S(\alpha, \beta)$ for $\mu=M, M+1, M+2$. Assume $\tilde{\theta}, \theta \in \mathbf{R}, \tilde{\theta}<\theta$, such that $(\tilde{\theta}, \theta)$ does not contain any Stokes number of level $1^{+}$of (1.1). Define $D:=D\left(\frac{1}{2}(M+1) ; \tilde{\theta}, \theta\right)$.

Assume we have fundamental systems $\left\{f_{l} u_{l}^{(\mu)}\right\}_{l=1}^{n}, \mu=M, M+1, M+2$ such that for all $l \in\{1, \ldots, n\}$ :

(i) $u_{l}^{(\mu)} \in\left(\mathcal{A}_{\left(1 / k_{1}\right)}\right)^{n}\left(\Gamma_{\mu}\right)[\log s], u_{l}^{(\mu)} \sim \hat{u}_{l}$ on $\Gamma_{\mu}$ if $\mu=M, M+1, M+2$,

(ii) $u_{l}^{(\mu+1)}-u_{l}^{(\mu)} \in\left(\mathcal{A}^{\leq-1^{+}}\right)^{n}\left(\Gamma_{\mu} \cap \Gamma_{\mu+1}\right)$ if $\mu=M, M+1$.

Then there exists a fundamental system $\left\{f_{l} \tilde{u}_{l}\right\}_{l=1}^{n}$ of equation (1.1) such that for all $l \in\{1, \ldots, n\}$ :

$$
\begin{gathered}
\tilde{u}_{l}-u_{l}^{(\mu)} \in\left(\mathcal{A}^{\leq-1}\right)^{n}\left(D \cap H_{\mu}\right) \text { if } \mu=M, M+2, \\
\tilde{u}_{l}-u_{l}^{(M+1)} \in\left(\mathcal{A}^{\leq-1^{+}}\right)^{n}\left(H_{M+1}\right) .
\end{gathered}
$$

Moreover, for each $l \in\{1, \ldots, n\}$ the function $\tilde{u}_{l} \in\left(\mathcal{A}_{\left(1 / k_{1}\right)}\right)^{n}(D)[\log s]$ is uniquely determined by these properties.

Remark. We can find $\alpha$ and $\beta$ satisfying the above conditions if and only if $[\nu \pi-\pi / 2, \nu \pi+\pi / 2]$ is not a Stokes interval of level 1 for any $\nu \in \mathbf{Z}$. This corresponds to the condition that $\Re b_{l} \neq \Re b_{m}$ if $b_{l} \neq b_{m}$.

Proof. Throughout the proof $l, m \in\{1, \ldots, n\}$. Let $h:=(M+1) / 2$, so $h$ is an integer. We will write $m \prec l$ if $f_{m l} \in \mathcal{A}^{\leq-1^{+}}\left(H_{2 h}\right)$, and $I(l):=\{m \mid m \prec$ $l\}$. The relation $\prec$ gives a partial ordering on $\{1, \ldots, n\}$. We will prove the proposition by induction with respect to this partial ordering.

Let $p_{m}^{(\mu)}, \mu=M, M+1$, be the 1-periodic analytic functions such that

$$
u_{l}^{(\mu+1)}-u_{l}^{(\mu)}=\sum_{m=1}^{n} p_{m}^{(\mu)} f_{m l} u_{m}^{(\mu)}, \mu=M, M+1 .
$$

As $u_{l}^{(\mu+1)}-u_{l}^{(\mu)} \in\left(\mathcal{A}^{\leq-1^{+}}\right)^{n}\left(\Gamma_{\mu} \cap \Gamma_{\mu+1}\right)$ by assumption, it follows from Lemma 7 that each of the summands must belong to this set. If $m \notin I(l)$ then $e^{d_{m l} s \log s}$ is unbounded on $H_{2 h}$, so $\Re d_{m l} s$ is positive on $H_{2 h}$. Therefore 
$f_{m l} \notin \mathcal{A}^{\leq-1^{+}}\left(\Gamma_{\mu} \cap \Gamma_{\mu+1}\right)$ for $\mu=M, M+1$ and Lemma 8 tells us that $p_{m}^{(\mu)}=0$. So in (7.8) we only need to sum over $m \in I(l)$.

First let $l$ be such that $I(l)=\emptyset$. Then $u_{l}^{(\mu)}$ is independent of $\mu \in\{M, M+$ $1, M+2\}$ and $\tilde{u}_{l}=u_{l}^{(\mu)}$ satisfies (7.6) and (7.7).

Now let $l$ be such that $\tilde{u}_{m}$ have been defined, and satisfy (7.6) and (7.7) for all $m \in I(l)$. The functions $f_{m} \tilde{u}_{m}, m \in I(l)$, together with the functions $f_{m} u_{m}^{(M+1)}, m \notin I(l)$, form a fundamental system of solutions. With the aid of Lemmas 7 and 8 we may conclude as above that

$$
u_{l}^{(M+1)}-u_{l}^{(M)}=\sum_{m \in I(l)} \tilde{p}_{m}^{(M)} f_{m l} \tilde{u}_{m},
$$

for some 1-periodic analytic functions $\tilde{p}_{m}^{(M)}$ on a neighbourhood of $\infty$ in $Q:=\Gamma_{M} \cap \Gamma_{M+1}$, and $\tilde{p}_{m}^{(M)} f_{m l} \in \mathcal{A}^{\leq-1^{+}}(Q)$. According to Lemma 19 the functions $\tilde{p}_{m}^{(M)}(s)$ can be written as $p_{m-}^{(M)}(s)+p_{m+}^{(M)}(s)$ with $p_{m-}^{(M)} f_{m l} \in$ $\mathcal{A}^{\leq-1}\left(D \cap H_{M}\right)$ and $p_{m+}^{(M)} f_{m l} \in \mathcal{A}^{\leq-1}\left(D \cap H_{M+2}\right) \cap \mathcal{A}^{\leq-1^{+}}\left(H_{M+1}\right)$. We define

$$
u_{l, 1}:=u_{l}^{(M)}+\sum_{m \in I(l)} p_{m-}^{(M)} f_{m l} \tilde{u}_{m}
$$

so that $u_{l, 1}-u_{l}^{(M)} \in\left(\mathcal{A}^{\leq-1}\right)^{n}\left(D \cap H_{M}\right)$ and

$$
\begin{aligned}
& u_{l, 1}-u_{l}^{(M+1)} \\
& =-\sum_{m \in I(l)} p_{m+}^{(M)} f_{m l} \tilde{u}_{m} \in\left(\mathcal{A}^{\leq-1}\right)^{n}\left(D \cap H_{M+2}\right) \cap\left(\mathcal{A}^{\leq-1^{+}}\right)^{n}\left(H_{M+1}\right) .
\end{aligned}
$$

From these relations and assumption (ii) it follows that $u_{l}^{(M+2)}-u_{l, 1} \in$ $\left(\mathcal{A}^{\leq-1^{+}}\right)^{n}\left(\Gamma_{M+1} \cap \Gamma_{M+2}\right)$ and as above we find that there exist 1-periodic functions $p_{m-}^{(M+1)}(s), p_{m+}^{(M+1)}(s), m \in I(l)$, such that

$$
u_{l}^{(M+2)}-u_{l, 1}=\sum_{m \in I(l)}\left(p_{m-}^{(M+1)} f_{m l} \tilde{u}_{m}+p_{m+}^{(M+1)} f_{m l} \tilde{u}_{m}\right),
$$

where the first term of each summand belongs to $\left(\mathcal{A}^{\leq-1}\right)^{n}\left(D \cap H_{M+2}\right)$, and the second one to $\left(\mathcal{A}^{\leq-1}\right)^{n}\left(D \cap H_{M}\right) \cap\left(\mathcal{A}^{\leq-1^{+}}\right)^{n}\left(H_{M+1}\right)$. Hence, if we define

$$
\tilde{u}_{l}:=u_{l}^{(M+2)}-\sum_{m \in I(l)} p_{m-}^{(M+1)} f_{m l} \tilde{u}_{m}
$$

then

$$
\tilde{u}_{l}=u_{l, 1}+\sum_{m \in I(l)} p_{m+}^{(M+1)} f_{m l} \tilde{u}_{m}
$$


and it is easy to verify that $\tilde{u}_{l}$ satisfies (7.6) and (7.7). The uniqueness of $\tilde{u}_{l}$ follows from Theorem 15 .

Theorem 18 can be proved similarly to Theorem 13, with the aid of Propositions 9,10 and 20 .

\section{References}

[Bal94] W. Balser, From Divergent Power Series to Analytic Functions, Lecture Notes in Mathematics 1582, Springer Verlag, Heidelberg, 1994.

[BBRS91] W. Balser, B.L.J. Braaksma, J.-P. Ramis and Y. Sibuya, Multisummability of formal power series solutions of linear ordinary differential equations, Asympt. Anal., 5 (1991), 27-45.

[BT33] G.D. Birkhoff and W.J. Trjitzinsky, Analytic theory of singular difference equations, Acta Math., 60 (1933), 1-89.

[Bra91] B.L.J. Braaksma, Multisummability and Stokes multipliers of linear meromorphic differential equations, J. Diff. Eq., 92 (1991), 45-75.

[Bra92] _ Multisummability of formal power series solutions of nonlinear meromorphic differential equations, Ann. Inst. Fourier, 42 (1992), 517-540.

[BF96] B.L.J. Braaksma and B.F. Faber, Multisummability for some classes of difference equations, Ann. Inst. Fourier, 46(1) (1996), 183-217.

[BH75] B.L.J. Braaksma and W.A. Harris, Jr., On an open problem in the theory of linear difference equations, Nieuw Archief voor Wiskunde (3), XXIII (1975), 228-240.

[BIS] B.L.J. Braaksma, G.K. Immink and Y. Sibuya, The Stokes phenomenon in exact asymptotics, Pacific J. Math., 187 (1999), 13-50.

[Eca85] J. Ecalle, Les fonctions résurgentes, III, Publ. Math. Orsay, 1985.

[Eca87] L'accélération des fonctions résurgentes, Manuscript, 1987.

[Fab97] B.F. Faber, Difference equations and summability, Revista del Seminario Iberoamericano de Matemáticas, V (1997), 53-63.

[Imm84] G.K. Immink, Asymptotics of Analytic Difference Equations, Lecture Notes in Mathematics, 1085, Springer Verlag, Heidelberg, 1984.

[Imm88] _ Asymptotic expansions with error bounds for solutions of difference equations of 'level $1^{+}$', Équations différentielles dans le champ complexe, Vol. 1, I.R.M.A., Strasbourg, (1988), 35-60.

[Imm91]_ Reduction to canonical forms and the Stokes phenomenon in the theory of linear difference equations, SIAM J. Math. Anal., 22 (1991), 238-259.

[Imm]_ On the summability of the formal solutions of a class of inhomogeneous linear difference equations, Funkcial. Ekvac., 39(3) (1996), 469-490.

[Imm96] _ A particular type of summability of divergent power series, with an application to linear difference equations, Preprint, 1996.

[Mal95] B. Malgrange, Sommation des séries divergentes, Expo. Math., 13 (1995), 163222.

[MR92] B. Malgrange and J.-P. Ramis, Fonctions multisommables, Ann. Inst. Fourier, 41 (1992), 353-368. 
[MR91] J. Martinet and J.-P. Ramis, Elementary acceleration and multisummability, Ann. Inst. H. Poincaré, Phys. Théor., 54 (1991), 331-401.

[RS94] J.-P. Ramis and Y. Sibuya, A new proof of multisummability of formal solutions of non-linear meromorphic differential equations, Ann. Inst. Fourier, 44 (1994), 811-848.

[Tov96] A.I. Tovbis, Multisummable solutions of nonlinear ordinary differential equations, J. Diff. Eq., 124 (1996), 359-377.

[vdPS97] M. van der Put and M.F. Singer, Galois theory of difference equations, Lecture Notes in Mathematics, 1666, Springer Verlag, Heidelberg, 1997.

Received October 22, 1998.

University of Groningen

Department of Mathematics, P.O.Box 800

9700 AV GRONINGEN

The NetherLands

E-mail address: B.L.J.Braaksma@math.rug.nl

UNIVERSITY OF GRONINGEN

Department of Mathematics, P.O.Box 800

9700 AV GRONINGEN

The Netherlands

E-mail address: bernard@math.rug.nl

University of Groningen

Department of Econometrics, P.O.Box 800

9700 AV GRONINGEN

The Netherlands

E-mail address: G.K.Immink@eco.rug.nl 